"A FEA e a USP respeitam os direitos autorais deste trabalho. Nós acreditamos que a melhor proteção contra o uso ilegítimo deste texto é a publicação online. Além de preservar o conteúdo motiva-nos oferecer à sociedade o conhecimento produzido no âmbito da universidade pública e dar publicidade ao esforço do pesquisador. Entretanto, caso não seja do interesse do autor manter o documento online, pedimos compreensão em relação à iniciativa e o contato pelo e-mail bibfea@usp.br para que possamos tomar as providências cabíveis (remoção da tese ou dissertação da BDTD)." 
UNIVERSIDADE DE SÃO PAULO

FACULDADE DE ECONOMIA, ADMINISTRAÇÃO E CONTABILIDADE INSTITUTO DE MATEMÁTICA E ESTATÍSTICA

MESTRADO PROFISSIONALIZANTE "MODELAGEM MATEMÁTICA EM FINANÇAS"

APREÇAMENTO DE OPÇÕES ASIÁTICAS E APLICAÇÃo AO MERCADO BRASILEIRO DE TAXAS DE CÂMBIO

\author{
Ivo Riemma Philipson
}

Orientador: Prof. Dr. Henrique von Dreifus

São Paulo

2002 


\section{APREÇAMENTO DE OPÇÕES ASIÁTICAS E APLICAÇÃO AO MERCADO BRASILEIRO DE TAXAS DE CÂMBIO}

Ivo Riemma Philipson

Dissertação apresentada à
Faculdade de Economia,
Administração e Contabilidade
e ao Instituto de Matemática e
Estatística da Universidade de
São Paulo para obtenção do
Título de Mestre, sob
orientação do Prof. Dr.
Henrique von Dreifus.

São Paulo 


\section{AGRADECIMENTOS}

Ao Prof. Dr. Henrique von Dreifus, pela sua orientação, sempre estimulando e indicando os caminhos, e pela amizade.

Ao Banco Bradesco S.A., na pessoa do Sr. José Carlos Luiz, pela confiança e apoio demonstrados durante todo o programa.

Aos professores do programa do Mestrado Profissionalizante Modelagem Matemática em Finanças, pela dedicação e auxílio durante todo o curso.

Aos meus pais, pelos exemplos e incentivos sempre demonstrados.

À minha esposa, pelo estímulo e paciência, e aos meus filhos pela compreensão e pela cessão do computador durante longos fins de semana.

Aos funcionários da Fundação Instituto de Pesquisas Econômicas, pelo atendimento sempre cordial e eficiente. 
"The journey, not the arrival, matters:

the voyage, not the landing"

Paul Theroux 


\section{RESUMO}

Esta dissertação compara o apreçamento de opções asiáticas através de dois métodos: o primeiro envolvendo a resolução numérica de uma EDP (Equação a Derivadas Parciais), e o segundo estabelecendo um intervalo suficientemente pequeno que contém o preço da opção. Ambos os métodos são analisados com relação aos erros envolvidos, ao tempo necessário para o cálculo, e às possibilidades de utilização prática. Apresentam-se exemplos de apreçamento de opções asiáticas com preço de exercício fixo aplicadas a taxas de câmbio, comparando-as com suas similares do tipo européias.

\section{ABSTRACT}

This dissertation compares the pricing of Asian options through two different methods: numerically solving a Partial Differential Equation (EDP) and setting a sufficiently small interval containing the option price. Both methods are analyzed concerning the errors involved, the time consumed and possibilities of practical use. Examples of exchange rate fixed strike Asian options are presented and compared with similar European ones. 
1. INTRODUÇÃO E DEFINIÇÕES .............................................................................. 1

2. APREÇAMENTO POR EDP ..............................................................................

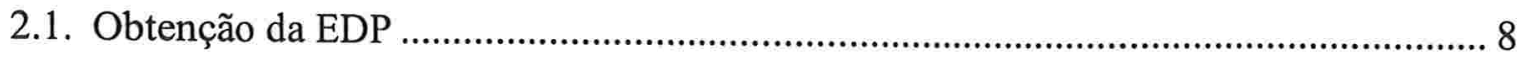

2.2. Implementação do Algoritmo de Resolução da EDP ............................................... 15

2.2.1. Montagem da Malha........................................................................................ 17

2.2.2. Implementação das Condições de Contorno …………………………………..... 18

2.2.3. Cálculo do Valor da Equação nos Nós Adjacentes ............................................. 18

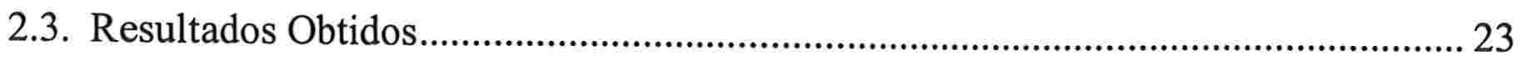

3. DETERMINAÇÃO DE LIMITES PARA O PREÇO DA OPÇÃO.............................. 28

3.1. Obtenção de Limite Inferior para o Preço da Opção................................................ 32

3.2. Obtenção Numérica do Limite ................................................................................. 36

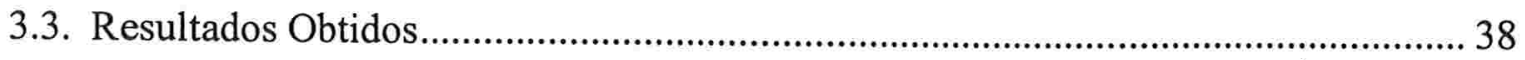

4. UMA APLICAÇÃO AO MERCADO BRASILEIRO DE TAXAS DE CÂMBIO .... 43

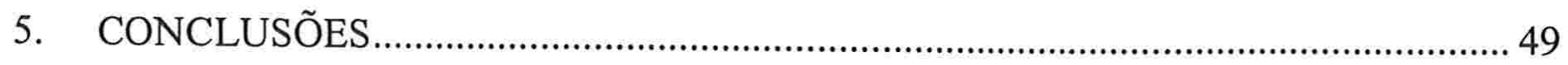

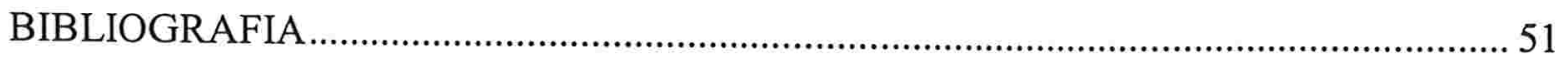




\section{INTRODUÇÃO E DEFINIÇÕES}

O mercado financeiro brasileiro, embora bem desenvolvido do ponto de vista tecnológico, é vulnerável em uma série de aspectos. Independentemente de quais sejam as causas desta vulnerabilidade, um dos efeitos mais imediatos e diretos que ela gera é a diminuição eventual da liquidez de grande parte dos instrumentos do mercado, fato que, por sua vez, muitas vezes inibe o apetite dos investidores por operações estruturadas de longo prazo. Embora, do ponto de vista dos investidores, exista a necessidade de proteção de ativos e passivos por períodos maiores (um ano ou mais), a questão da liquidez leva-os, em geral, a optar por operações de menor prazo (tipicamente poucos meses), que são refeitas periodicamente, próximo a cada vencimento, e enquanto houver a necessidade de proteção. As consequêencias desta estratégia são bastante óbvias: por um lado, a proteção pelo período mais longo não é efetiva, expondo os ativos e passivos a riscos de flutuação dos preços durante os períodos de renovação; por outro, os custos envolvidos nas constantes renovações são muito elevados, podendo inclusive inviabilizá-las. Duas alternativas possiveis, e que muitas vezes são utilizadas, contribuindo para a eliminação dos dois pontos levantados, são:

- a celebração de um contrato de opções tradicional, com maturidade compatível com o período pelo qual se deseja manter a proteção, e no qual o investidor se protege de variações indesejadas no preço do ativo relevante para a sua posição; e

- a celebração de um contrato de swap, também com maturidade compatível com o período pelo qual se deseja manter a proteção, e no qual o investidor se protege de variações no preço de um ativo em relação ao preço de outro ativo.

Estas estratégias, no entanto, embutem novos riscos, em parte também oriundos da falta de liquidez dos mercados. No caso de uma opção tradicional, seu payoff depende do preço do ativo-base (o ativo sobre o qual a opção é definida) em uma única data, pré-determinada, e, portanto, está sujeito às flutuações de preço que venham a ocorrer nessa data. Já no caso de um swap, o acerto dos fluxos financeiros na maturidade do contrato é realizado com base 
nas variações dos preços de cada um dos dois ativos envolvidos na operação, podendo gerar resultados diferentes dos efetivamente desejados.

É neste contexto que o presente trabalho pretende oferecer uma contribuição, ao abordar o tema de opções asiáticas, uma classe de opções que têm em comum uma característica em relação a seu payoff: a dependência da média dos preços do ativo-base em um determinado periodo.

A idéia de utilizar uma média de preços na determinação do payoff, em contraposição ou em complemento à utilização de um único preço coletado em uma data pré-determinada, vem de encontro à busca de uma solução para os problemas levantados acima: por se tratar de uma média, a influência do preço tomado em uma determinada data é diluída, tornando a média em uma quantidade relativamente imune às variações bruscas de preço constatadas em pequenos intervalos de tempo.

O termo "asiáticas" é utilizado na denominação desta classe de opções, segundo Campbell, Lo e MacKinlay (1997), por elas terem surgido originalmente nos mercados asiáticos, tipicamente de pequena capitalização se comparados com mercados mais desenvolvidos da Europa e Estados Unidos e, por esse motivo, potencialmente mais sujeitos a manipulações de preços. Já segundo Vorst (in Nelken, 1996), esta denominação originou-se entre os funcionários do Bankers Trust, que vendia este tipo de opções a firmas japonesas que queriam se proteger contra flutuações da taxa de câmbio do yen em relação a outras moedas. Os primeiros contratos de opções desta classe surgiram no final dos anos 70, e simplesmente substituíam o preço do ativo-objeto no vencimento do contrato pela média dos preços observados durante toda a vida da opção, mantendo fixo o preço de exercício da opção. Este tipo de opção asiática é ainda hoje um dos mais populares, embora outros tipos tenham surgido posteriormente. A partir de meados da década de 80 , estes contratos se tornaram bastante populares, sendo negociados, em geral, fora dos pregões, no que é denominado mercado de balcão. 
De maneira geral, as opções asiáticas podem ser classificadas segundo o tipo de média e segundo o tipo de payoff, da seguinte forma:

- Tipos de média principais

- Aritmética

$$
I=\frac{1}{N} \sum_{i=1}^{N} w_{i} S_{i},
$$

onde $N$ é o número de elementos que compõem a média, e os $w_{i}$ são pesos tais que

$$
\sum_{i=1}^{N} w_{i}=N
$$

- Geométrica

$$
I=\left(\prod_{i=1}^{N} w_{i} S_{i}\right)^{1 / p}
$$

onde $N$ é o número de elementos que compõem a média, e os $w_{i}$ são pesos tais que

$$
\prod_{i=1}^{N} w_{i}=P
$$

- Tipos de payoff

Nas especificações abaixo, $S_{T}$ é o preço do ativo-base na maturidade, $X$ é o preço de exercício (fixo), e $I$ é a média:

- Opção de compra com preço de exercício fixo (conhecida como fixed strike call, ou average rate call)

$$
\max (I-X, 0)
$$


- Opção de venda com preço de exercício fixo (conhecida como fixed strike put, ou average rate put)

$$
\max (X-I, 0)
$$

- Opção de compra com preço de exercício variável (conhecida como floating strike call, ou average strike call)

$$
\max \left(S_{T}-I, 0\right)
$$

- Opção de venda com preço de exercício variável (conhecida como floating strike put, ou average strike put)

$$
\max \left(I-S_{T}, 0\right)
$$

Além destas classificações, alguns autores (como Wilmott, 2000) consideram importante a forma de coleta de dados, que pode ser discreta (apenas preços específicos são considerados, sendo os mais comuns o preço médio ou o de fechamento) ou contínua (todos os negócios são considerados). Também a periodicidade, no caso da coleta discreta, é relevante, usualmente variando entre diária, semanal, ou mensal. Na prática, a utilização de médias de preços coletados continuamente é inviável, por questões de confiabilidade dos dados ou mesmo de regulamentação dos mercados, mas esta abordagem, como limite de um processo de coleta discreto em um período relativamente longo, é muito útil no processo de apreçamento de opções asiáticas.

A introdução da média na determinação do payoff pode reduzir significativamente o preço inicial da opção, quando comparado com o preço de uma opção tradicional com todas as outras características iguais. Este é um dos fatores que contribui para tornar as opções asiáticas bastante populares nos mercados em que elas são negociadas. É fácil compreender heuristicamente, no exemplo a seguir, porque isso acontece. 
Exemplo 1.1: Seja $C A$ o preço de uma opção asiática de compra com preço de exercício fixo $X$ e maturidade $T$, e $C E$ o preço de uma opção européia sobre o mesmo ativo-base, com o mesmo preço de exercício $X$ e de mesma maturidade $T$. Na maturidade, temos:

$$
C A_{T}=\max \left(I_{T}-X, 0\right) \quad \text { e } \quad C E_{T}=\max \left(S_{T}-X, 0\right),
$$

onde $I_{T}$ é a média de preços do ativo-base no período $[0 \ldots T]$ e $S_{T}$ é o preço do ativo-base na maturidade $(T)$. Se, no instante $T-1$, tivéssemos $I_{T-1}=S_{T-1}$, então, para qualquer valor que $S$ viesse a assumir no instante $T$, teríamos sempre $\left|I_{T}-I_{T-1}\right| \leq\left|S_{T}-S_{T-1}\right|$, significando que a variação da média é sempre menor ou igual à variação do preço do ativo-base, o que implica em $\mathrm{E}_{T-1}\left(C A_{T}\right) \leq \mathrm{E}_{T-1}\left(C E_{T}\right)$. Andando sucessivamente para trás no tempo, e utilizando o mesmo raciocínio, obtemos, para todo instante $\tau$ em $[0 \ldots T-2]$, que $\mathrm{E}_{\tau}\left(C A_{T}\right) \leq \mathrm{E}_{\tau}\left(C E_{T}\right)$. Em particular, no instante $\tau=0$, teríamos $\mathrm{E}_{0}\left(C A_{T}\right) \leq \mathrm{E}_{0}\left(C E_{T}\right)$. Por outro lado, supondo que o instante $\tau=0$ é o instante da primeira coleta de dados para compor a média $I$, temos $I_{0}=S_{0}$, implicando em $C A_{0} \leq C E_{0}$. Como vimos acima, isto se deve ao fato de que a variação da média, à medida que a ela vão sendo incorporados novos preços, é sempre menor ou igual à variação do preço do ativo-base (e só é igual em dois casos particulares: se a média que buscamos for composta por um único preço, caso em que a média é sempre igual ao preço do ativo-base, e a opção asiática se transforma em uma opção européia tradicional; ou se os preços forem constantes, caso em que os payoffs das duas opções são iguais). É importante ressaltar que o raciocínio exposto acima, para um instante $\tau$ qualquer, só vale quando $I_{\tau}=S_{\tau}$. Mas como, no instante $\tau=0$, sempre vale que $I_{0}=S_{0}$, podemos afirmar que o preço inicial de uma opção asiática é sempre menor ou igual ao preço da opção européia equivalente. A magnitude da diferença dos preços entre essas duas opções é uma função da volatilidade do ativo-base, da maturidade das opções e da taxa de juros livre de risco, bem como do tipo de média que é utilizada, e é um dos assuntos que serão discutidos nos capítulos seguintes.

Os capítulos seguintes deste trabalho estão organizados da seguinte forma: 
Capítulo 2: aborda a questão do apreçamento de uma opção asiática. A partir da dedução dos parâmetros relevantes, e de uma Equação a Derivadas Parciais (EDP) nesses parâmetros, desenvolve-se um algoritmo numérico para estimar o preço da opção, através do método explícito, e discute-se os erros envolvidos neste processo;

Capitulo 3: propõe a estimação de um intervalo de valores que contenha o preço da opção, de uma maneira computacionalmente mais eficiente do que a resolução da EDP. Discute-se ainda a eficácia deste método, em termos da amplitude do intervalo obtido, do tempo demandado para o cálculo, e dos erros envolvidos no processo;

Capítulo 4: apresenta exemplos práticos no mercado brasileiro, juntamente com uma discussão sobre possíveis vantagens e desvantagens da utilização destas opções, quando comparadas com opções européias tradicionais;

Capitulo 5: apresenta as principais conclusões, bem como sugestões para novos trabalhos sobre o tema desenvolvido, visando à implementação das opções asiáticas no mercado financeiro brasileiro. 


\section{APREÇAMENTO POR EDP}

O estudo matemático do apreçamento de opções asiáticas revela alguns aspectos interessantes, caracterizados pelas diferenças em relação às opções tradicionais. Enquanto estas últimas têm seu apreçamento dependente apenas do valor do ativo-objeto na maturidade, as opções asiáticas têm seu preço dependente do caminho (história) traçado pelo ativo-objeto durante um certo período, anterior à maturidade. O caminho em questão é representado, na fórmula do payoff da opção, por algum tipo de média. Esta média é um processo estocástico, que exerce um papel similar ao do preço do ativo-objeto em uma opção tradicional, mas que tem características diversas com relação à sua evolução no tempo: enquanto o preço obedece a uma distribuição log-normal, o mesmo em geral não acontece com a média. Por exemplo, assumindo uma distribuição log-normal para o preço $S$ do ativo-base, temos

$$
S_{t}=S_{t-1} e^{\left(\left(r-\frac{1}{2} \sigma^{2}\right) d t+\sigma d B_{t}\right)}=S_{0} e^{\left(\left(r-\frac{1}{2} \sigma^{2}\right) d t+\sigma d B_{0}\right)} e^{\left(\left(r-\frac{1}{2} \sigma^{2}\right) d t+\sigma d B_{1}\right)} \ldots e^{\left(\left(r-\frac{1}{2} \sigma^{2}\right) d t+\sigma d B_{t}\right)}
$$

o que implica em

$$
d S_{t}=\left(r-\frac{1}{2} \sigma^{2}\right) S_{t} d t+\sigma S_{t} d B_{t}, \text { onde }
$$

$B_{t}$ é um processo browniano padrão, cujos incrementos são não correlacionados;

$r$ é a taxa de juros livre de risco, assumida constante;

$\sigma$ é a variância dos retornos, também assumida constante.

A média aritmética $A$ dos preços (tomados de forma discreta) fica

$$
\begin{aligned}
A & =\frac{1}{T} \sum_{t=i}^{T} S_{t}=\frac{1}{T}\left(S_{0}+S_{0} e^{\left(\left(r-\frac{1}{2} \sigma^{2}\right) d t+\sigma d B_{0}\right)}+\ldots+S_{0} e^{\left(\left(r-\frac{1}{2} \sigma^{2}\right) d t+\sigma d B_{0}\right)} e^{\left(\left(r-\frac{1}{2} \sigma^{2}\right) d t+\sigma d B_{1}\right)} \ldots e^{\left(\left(r-\frac{1}{2} \sigma^{2}\right) d t+\sigma d B_{T-1}\right)}\right) \\
& =\frac{1}{T} S_{t}\left(1+e^{\left(r-\frac{1}{2} \sigma^{2}\right) d t+\sigma d B_{0}}+e^{\left(r-\frac{1}{2} \sigma^{2}\right) d t+\sigma d B_{0}} e^{\left(r-\frac{1}{2} \sigma^{2}\right) d t+\sigma d B_{1}}+\ldots+e^{\left(r-\frac{1}{2} \sigma^{2}\right) d t+\sigma d B_{0}} e^{\left(r-\frac{1}{2} \sigma^{2}\right) d t+\sigma d B_{1}} \ldots e^{\left(r-\frac{1}{2} \sigma^{2}\right) d t+\sigma d B_{\tau}-1}\right)
\end{aligned}
$$

que não tem uma distribuição de probabilidade conhecida. 
Propriedades como a descrita acima tornam não trivial a tarefa de apreçar uma opção asiática por Equações a Derivadas Parciais (EDP), em contraste com a tarefa de apreçar opções tradicionais por EDP, já amplamente revisitada em inúmeros trabalhos, desde a publicação do trabalho seminal de Black e Scholes em 1973, e motivando o desenvolvimento a seguir.

\subsection{Obtenção da EDP}

Entre diversos trabalhos em que a tarefa de obtenção da EDP para opções asiáticas é desenvolvida, optamos por nos ater ao artigo de Rogers e Shi (1995), cuja metodologia é também utilizada por Klebaner (1999, p.271), e que, a partir de conceitos simples de cálculo estocástico, obtém os resultados que buscamos. Esta metodologia é reproduzida a seguir, com mais detalhes, para permitir o seu fácil entendimento.

Uma opção asiática de compra, com preço de exercício fixo $X$ (fixed strike) e maturidade $T$, tem seu payoff $C(T)$ definido por

$$
C(T)=\left(\frac{1}{T} \int_{0}^{T} S(u) d u-X\right)^{+}
$$

Seu preço no instante $t(0 \leq t \leq T)$ é dado por

$$
C(t)=e^{-r(T-t)} \mathrm{E}\left[\left(\frac{1}{T} \int_{0}^{T} S(u) d u-X\right)^{+} \mid \Im_{t}\right], \text { onde }
$$

$r$ é a taxa de juros livre de risco,

e $\mathfrak{I}_{t}$ é o conjunto (conhecido) dos preços do ativo-base no intervalo $[0, t]$. 
Para nos auxiliar no processo de apreçamento da opção, vamos definir uma função $\Phi(t, x)$ da seguinte forma:

$$
\Phi(t, x) \equiv \mathrm{E}\left[\left(\frac{1}{T} \int_{t}^{T} S(u) d u-x\right)^{+} \mid S(t)=1\right],
$$

e vamos desenvolver um processo martingale $M(t)$ para o preço da opção, relacionando-o com esta função:

$$
\begin{aligned}
M(t) & \equiv \mathrm{E}\left[\left(\frac{1}{T} \int_{0}^{T} S(u) d u-X\right)^{+} \mid \Im_{t}\right] \\
& =\mathrm{E}\left[\left(\frac{1}{T} \int_{0}^{t} S(u) d u+\frac{1}{T} \int_{t}^{T} S(u) d u-X\right)^{+} \mid \Im_{t}\right] \\
& =\mathrm{E}\left[\left(\frac{1}{T} \int_{t}^{T} S(u) d u-\left(X-\frac{1}{T} \int_{0}^{t} S(u) d u\right)\right)^{+} \mid \Im_{t}\right] \\
& =S(t) \mathrm{E}\left[\left(\frac{1}{T} \int_{t}^{T} \frac{S(u)}{S(t)} d u-\frac{X-\frac{1}{T} \int_{0}^{t} S(u) d u}{S(t)}\right)^{+} \mid \Im_{t}\right] \\
& =S(t) \phi(t, Y(t, S(t))),
\end{aligned}
$$

onde

$$
Y(t, S(t)) \equiv \frac{X-\frac{1}{T} \int_{0}^{t} S(u) d u}{S(t)} .
$$

Aplicando a Fórmula de Itô em $Y(t, S(t))$, e fazendo $Y(t, S(t))=Y$ e $S(t)=S$, obtemos 


$$
d Y=\frac{\partial Y}{\partial t} d t+\frac{\partial Y}{\partial S} d S+\frac{1}{2} \frac{\partial^{2} Y}{\partial S^{2}} d S^{2} .
$$

Mas

$$
\begin{aligned}
\frac{\partial Y}{\partial t} & =\frac{1}{S}\left(-\frac{1}{T} S\right)=-\frac{1}{T}, \\
\frac{\partial Y}{\partial S} & =-\frac{1}{S^{2}}\left(X-\frac{1}{T} \int_{0}^{t} S(u) d u\right)=-\frac{1}{S} Y, \\
e \frac{\partial^{2} Y}{\partial S^{2}} & =-\left(\frac{-2}{S^{3}}\left(X-\frac{1}{T} \int_{0}^{t} S(u) d u\right)\right)=\frac{2}{S^{2}} Y .
\end{aligned}
$$

Logo,

$$
d Y=-\frac{1}{T} d t-\frac{1}{S} Y d S+\frac{1}{S^{2}} Y d S^{2} .
$$

Agora, assumindo que o ativo-base obedece a uma distribuição log-normal, temos que

$$
d S=r S d t+\sigma S d B
$$

$\mathrm{e}$

$$
d S^{2}=\sigma^{2} S^{2} d B^{2}=\sigma^{2} S^{2} d t .
$$

Então podemos reescrever $d Y$ como 


$$
\begin{aligned}
d Y & =-\frac{1}{T} d t-\frac{1}{S} Y(r S d t+\sigma S d B)+\frac{1}{S^{2}} Y\left(\sigma^{2} S^{2} d t\right) \\
& =-\frac{1}{T} d t-Y r d t-Y \sigma d B+Y \sigma^{2} d t \\
& =\left(\left(\sigma^{2}-r\right) Y-\frac{1}{T}\right) d t-Y \sigma d B .
\end{aligned}
$$

Da definição de $\Phi$ é fácil ver que $\Phi$ é contínua (por partes), decrescente em $t$, e decrescente em $x$. Assumindo que $\Phi$ é suficientemente suave, podemos aplicar a fórmula de Itô para obter

$$
d \Phi=\frac{\partial \Phi}{\partial t} d t+\frac{\partial \Phi}{\partial Y} d Y+\frac{1}{2} \frac{\partial^{2} \Phi}{\partial Y^{2}} d Y^{2}
$$

Mas, como já vimos,

$$
d Y=\left(\left(\sigma^{2}-r\right) Y-\frac{1}{T}\right) d t-\sigma Y d B
$$

logo

$$
d Y^{2}=\sigma^{2} Y^{2} d t
$$

o que nos leva a

$$
\begin{aligned}
d \Phi & =\frac{\partial \Phi}{\partial t} d t+\frac{\partial \Phi}{\partial Y}\left(\left(\left(\sigma^{2}-r\right) Y-\frac{1}{T}\right) d t-\sigma Y d B\right)+\frac{1}{2} \frac{\partial^{2} \Phi}{\partial Y^{2}} \sigma^{2} Y^{2} d t \\
& =\left[\frac{\partial \Phi}{\partial t}+\frac{\partial \Phi}{\partial Y}\left(\left(\sigma^{2}-r\right) Y-\frac{1}{T}\right)+\frac{1}{2} \frac{\partial^{2} \Phi}{\partial Y^{2}} \sigma^{2} Y^{2}\right] d t-\frac{\partial \Phi}{\partial Y} \sigma Y d B
\end{aligned}
$$

Finalmente, em relação a $M(t)$, temos que 


$$
\begin{aligned}
d M & =\Phi d S+S d \Phi+d S d \Phi=\Phi d S+d \Phi(S+d S) \\
& =\Phi(r S d t+\sigma S d B)+\left(\left[\frac{\partial \Phi}{\partial t}+\frac{\partial \Phi}{\partial Y}\left(\left(\sigma^{2}-r\right) Y-\frac{1}{T}\right)+\frac{1}{2} \frac{\partial^{2} \Phi}{\partial Y^{2}} \sigma^{2} Y^{2}\right] d t-\frac{\partial \Phi}{\partial Y} \sigma Y d B\right)(S+r S d t+\sigma S d B) \\
& =S\left[\Phi r+\frac{\partial \Phi}{\partial t}+\frac{\partial \Phi}{\partial Y}\left(\left(\sigma^{2}-r\right) Y-\frac{1}{T}\right)+\frac{1}{2} \frac{\partial^{2} \Phi}{\partial Y^{2}} \sigma^{2} Y^{2}-\frac{\partial \Phi}{\partial Y} \sigma^{2} Y\right] d t+S\left[\Phi \sigma-\frac{\partial \Phi}{\partial Y} \sigma Y\right] d B
\end{aligned}
$$

e como, por definição, $M(t)$ é um martingale, concluímos que

$$
\Phi r+\frac{\partial \Phi}{\partial t}+\frac{\partial \Phi}{\partial Y}\left(\left(\sigma^{2}-r\right) Y-\frac{1}{T}\right)+\frac{1}{2} \frac{\partial^{2} \Phi}{\partial Y^{2}} \sigma^{2} Y^{2}-\frac{\partial \Phi}{\partial Y} \sigma^{2} Y=0,
$$

ou seja,

$$
\Phi r+\frac{\partial \Phi}{\partial t}-\frac{\partial \Phi}{\partial Y}\left(r Y+\frac{1}{T}\right)+\frac{1}{2} \frac{\partial^{2} \Phi}{\partial Y^{2}} \sigma^{2} Y^{2}=0
$$

Agora, definindo

$$
f(t, x) \equiv e^{-r(T-t)} \Phi(t, x),
$$

temos que o preço da opção pode ser escrito como

$$
C(t)=S(t) f(t, x)
$$

e a condição de contorno fica

$$
\begin{aligned}
f(T, x) & =\Phi(T, x)=\left(\frac{1}{T} \int_{T}^{T} \frac{S(u)}{S(T)} d u-x\right)^{+} \\
& =(-x)^{+} \\
& =x^{-} .
\end{aligned}
$$


No caso de uma opção asiática de compra, com preço de exercício igual ao preço do ativobase na maturidade (floating strike), o payoff é definido como

$$
\left(\frac{1}{T} \int_{0}^{T} S(u) d u-S(T)\right)^{+}
$$

Definimos então

$$
\Psi(t, x) \equiv \mathrm{E}\left[\left(\frac{1}{T} \int_{t}^{T} S(u) d u-S(T)-x\right)^{+} \mid S(T)=1\right],
$$

e desenvolvemos um processo martingale

$$
\begin{aligned}
M(t) & \equiv \mathrm{E}\left[\left(\frac{1}{T} \int_{0}^{T} S(u) d u-S(T)\right)^{+} \mid \Im_{t}\right] \\
& =\mathrm{E}\left[\left(\frac{1}{T} \int_{t}^{T} S(u) d u-S(T)-\left(-\frac{1}{T} \int_{0}^{T} S(u) d u\right)\right)^{+} \mid \Im_{t}\right] \\
& =S(t) \cdot \mathrm{E}\left[\left(\frac{1}{T} \int_{t}^{T} \frac{S(u)}{S(t)} d u-\frac{S(T)}{S(t)}-\left(-\frac{1}{T} \int_{0}^{T} \frac{S(u)}{S(t)} d u\right)\right)^{+} \mid \Im_{t}\right] \\
& =s(t) \Psi(t, Y(t, S(t))),
\end{aligned}
$$

onde

$$
Y(t, S(t))=\frac{1}{T} \int_{0}^{T} \frac{S(u)}{S(t)} d u,
$$

que é igual à função obtida no caso anterior (fixed strike), tomando $X=0$. Aplicando a fórmula de Itô, obtemos (como no caso anterior) 


$$
d Y=\left(\left(\sigma^{2}-r\right)-\frac{1}{T}\right) d t-Y \sigma d B,
$$

o que nos leva trivialmente a

$$
d \Phi=d \Psi
$$

e, definindo

$$
g(t, x)=e^{-r(T-t)} \Psi(t, x)
$$

obtemos a condição de contorno

$$
\begin{aligned}
g(T, x) & =\Psi(T, x) \\
& =\left(\frac{1}{T} \int_{T}^{T} \frac{S(u)}{S(T)} d u-\frac{S(T)}{S(T)}-x\right)^{+} \\
& =(-1-x)^{+} \\
& =(1+x)^{-} .
\end{aligned}
$$

Já no caso de uma opção asiática de compra com preço de exercício igual à média dos preços do ativo-base (random strike, conforme definido por Klebaner 1999, p. 254), e maturidade $T$, o payoff é definido como

$$
\left(S(T)-\frac{1}{T} \int_{0}^{T} S(u) d u\right)^{+},
$$

que é o oposto do payoff da opção floating strike definida anteriormente, e é igual ao payoff de uma opção asiática de venda do tipo floating strike. Então, trivialmente, obtemos a condição de contorno 


$$
g(T, x)=(1+x)^{+}
$$

É importante observar que, nos três casos explorados até aqui, a EDP relevante para o apreçamento da opção é a mesma. Podemos estender os resultados obtidos também para as opções asiáticas de venda, obtendo, para todos os tipos, a mesma EDP, mas com condições de contorno diferentes. Sintetizamos a seguir essas condições de contorno para três tipos de opções asiáticas de venda:

- Fixed strike

$$
\text { payoff: }\left(X-\frac{1}{T} \int_{0}^{T} S(u) d u\right)^{+}
$$

Condição de contorno: $f(T, x)=x^{+}$.

- Floating strike

$$
\text { payoff: }\left(S(T)-\frac{1}{T} \int_{0}^{T} S(u) d u\right)^{+}
$$

Condição de contorno: $f(T, x)=(1+x)^{+}$.

- Random strike

$$
\text { payoff: }\left(\frac{1}{T} \int_{0}^{T} S(u) d u-S(T)\right)^{+}
$$

(igual ao payoff de uma opção asiática de compra do tipo floating strike)

Condição de contorno: $f(T, x)=(1+x)^{-}$.

\subsection{Implementação do Algoritmo de Resolução da EDP}

Vimos, na seção anterior, que o preço $C$ de uma opção asiática pode ser escrito como: 


$$
C(t)=S(t) e^{-r(T-t)} \Phi(t, Y), \text { onde }
$$

$S(t)$ é o preço do ativo-base no instante t,

$r \quad$ é a taxa de juros livre de risco,

$T$ é a maturidade da opção, e

$\Phi(t, Y)$ é uma função tal que

$$
\Phi r+\frac{\partial \Phi}{\partial t}-\frac{\partial \Phi}{\partial Y}\left(r Y+\frac{1}{T}\right)+\frac{1}{2} \frac{\partial^{2} \Phi}{\partial Y^{2}} \sigma^{2} Y^{2}=0
$$

com a condição de contorno apropriada, que depende da definição do payoff da opção. Nosso objetivo é determinar o valor da opção, $C(t)$, em qualquer instante $t$, e para isso, vimos que basta determinarmos o valor de $\Phi(t, Y)$. A abordagem mais simples para este problema é a utilização de um método numérico. Existem muitos métodos numéricos, mais ou menos sofisticados, que poderiam ser utilizados, mas optamos pelo mais simples, conhecido como método de diferenças finitas explícito.

O método de diferenças finitas consiste dos seguintes passos:

- Montar uma malha;

- Definir o valor da equação nos extremos da malha (condições de contorno);

- Calcular o valor da equação nos nós adjacentes, a partir dos valores conhecidos;

- Terminar quando atingir o nó que se deseja calcular.

A forma de calcular o valor da equação nos nós adjacentes é o que caracteriza qual método de diferenças finitas está sendo utilizado. No caso do método explícito, esse valor é calculado a partir de três valores adjacentes. Veremos o método utilizado, com mais detalhes, a seguir. 


\subsubsection{Montagem da Malha}

A partir das duas variáveis relevantes no problema, o tempo e a quantidade $Y$, relacionada à média dos preços do ativo em cada instante, construímos uma malha bi-dimensional, particionando-a em $I$ intervalos de tamanho $\delta y$ para a quantidade $Y$, e $K$ intervalos de tamanho $\delta t$ para o tempo $T$. Além disso, como a quantidade $Y$ pode assumir valores positivos e negativos, definimos o seu intervalo de variação centrado em relação à razão $\frac{X}{S_{0}}$, onde $X$ é o preço de exercício da opção, variando de $\frac{X}{S_{0}}(1-\lambda)$ a $\frac{X}{S_{0}}(1+\lambda)$, e $\lambda$ é o fator de amplitude do intervalo. Definimos, também, o índice do tempo como decrescente, de maneira que o índice $k$ represente o instante $(T-k)$, e a maturidade da opção seja representada por $k=0$.

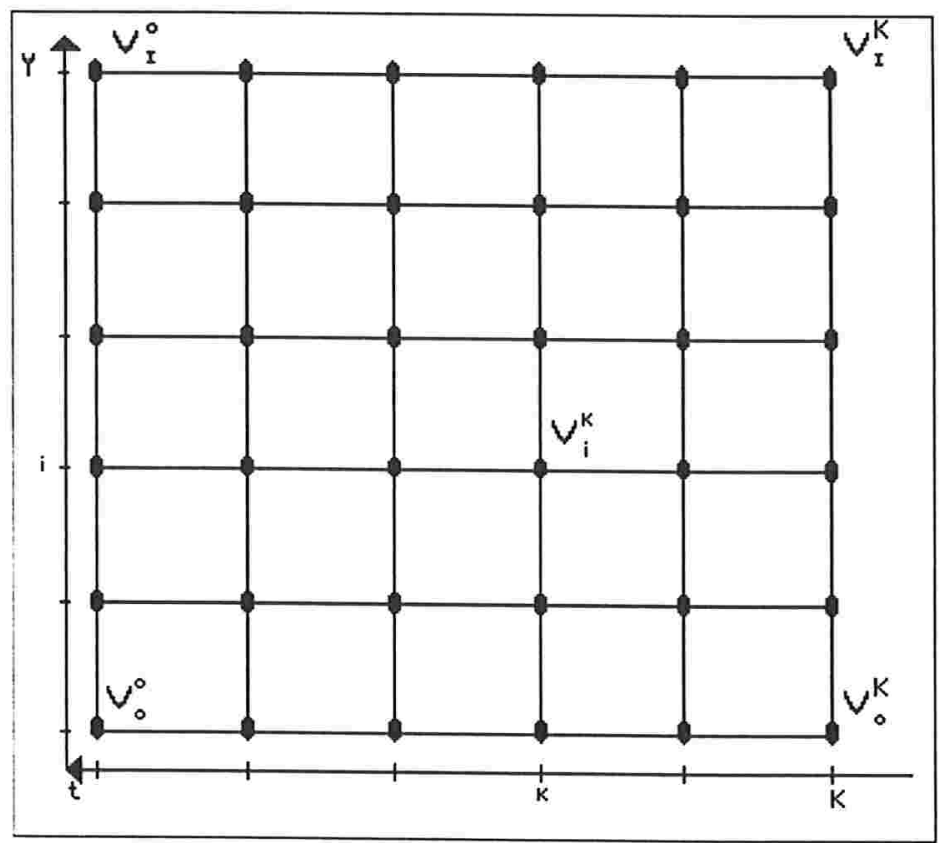

Figura 2.1: Um exemplo de malha para o algoritmo de resolução numérica.

Em seguida, definimos $V_{i}^{k}$ como o valor de $\Phi$ no nó $(k, i)$ da malha, $(0 \leq k \leq K)$ e $(0 \leq i \leq I)$, o que significa que $V_{i}^{k}$ é uma representação do valor de $\Phi$ quando $t=(T-k \delta t)$ e $Y=X / S_{0}(1-\lambda)+i \delta y$, como ilustrado na Figura 2.1. 


\subsubsection{Implementação das Condições de Contorno}

Em nosso problema, o payoff da opção é conhecido. Isto significa que sabemos o valor de $\Phi$ no instante $k=0$, para qualquer valor de $i$. Tal valor é dado por

$$
\Phi=V_{i}^{0}=\operatorname{payoff}(i \delta y)
$$

onde $\delta y$ é o passo de $Y$. Por exemplo, no caso da opção de compra com preço de exercício fixo (fixed strike call), temos

$$
V_{i}^{0}=(-i \delta y)^{+} \text {, para todo } 0 \leq i \leq I
$$

Já para os valores extremos relacionados à quantidade $Y$, nos instantes $t<T$, veremos na seção seguinte o procedimento adotado. Por ora, vamos apenas comentar que a definição destes valores depende do tipo de opção que estamos tratando. O valor da opção relacionado ao valor extremo inferior de $Y, V_{0}^{k}$, deve ser uma boa aproximação, para cada valor de $k$, do valor da opção quando $Y \rightarrow-\infty$, e, de maneira similar, no caso do valor extremo superior de $Y$, o valor da opção $V_{I}^{k}$ deve ser uma boa aproximação para o valor da opção quando $Y \rightarrow+\infty$.

\subsubsection{Cálculo do Valor da Equação nos Nós Adjacentes}

A partir da definição dos valores de $\Phi$ na maturidade da opção, vamos determinar os nós adjacentes, retroagindo no tempo. Os nós adjacentes são os nós $(i, k+1)$ tais que $V_{i}^{k}$ são conhecidos $(0 \leq i \leq I)$, o que significa que vamos retroagir no tempo, a cada passo $\delta t$, do instante $T-k$ para o instante $T-(k+1)$. Observando a EDP de $\Phi(t, Y)$, notamos que, para 
determinar o valor de $V_{i}^{k+1}$, precisamos determinar o valor de três derivadas parciais de $\Phi$ : a primeira derivada em relação ao tempo $\left(\frac{\partial \Phi}{\partial t}\right)$, e as duas primeiras derivadas em relação à quantidade $Y\left(\frac{\partial \Phi}{\partial Y} e \frac{\partial^{2} \Phi}{\partial Y^{2}}\right)$.

Os métodos de diferenças finitas têm este nome por aproximarem o valor de uma derivada por uma diferença (finita). Em nosso caso, vamos aproximar o valor das derivadas parciais utilizando valores adjacentes na malha que definimos. No caso de $\left(\frac{\partial \Phi}{\partial t}\right)$, temos

$$
\frac{\partial \Phi}{\partial t}(t, Y) \equiv \lim _{h \rightarrow 0} \frac{\Phi(t+h, Y)-\Phi(t, Y)}{h}
$$

e vamos calcular uma aproximação deste valor por

$$
\frac{\partial \Phi}{\partial t}(t, Y) \approx \frac{V_{i}^{k}-V_{i}^{k+1}}{\delta t}
$$

Ao procedermos desta forma, estamos introduzindo um erro em nosso cálculo, e para determinarmos a magnitude deste erro, vamos utilizar uma expansão em série de Taylor de $\Phi$ em torno do ponto $(t-\delta t, Y)$ :

$$
\Phi(t-\delta t, Y)=\Phi(t, Y)-\delta t \frac{\partial \Phi}{\partial t}(t, Y)+O\left(\delta t^{2}\right)
$$

Na malha que estamos utilizando, esta expansão é representada como

$$
V_{i}^{k}=V_{i}^{k+1}+\delta t \frac{\partial V_{i}^{k+1}}{\partial t}+O\left(\delta t^{2}\right)
$$


que, rearranjando, fica

$$
\frac{\partial V_{i}^{k+1}}{\partial t}=\frac{V_{i}^{k}-V_{i}^{k+1}}{\partial t}+O(\delta t)
$$

Ou seja, a magnitude do erro introduzido pela aproximação de $\frac{\partial \Phi}{\partial t}$ pela diferença acima é da ordem de $\delta t$.

Para $\frac{\partial \Phi}{\partial Y}$ vamos utilizar uma aproximação um pouco melhor, envolvendo os nós $V_{i+1}^{k} \mathrm{e}$ $V_{i-1}^{k}$, chamada de diferença central:

$$
\frac{\partial \Phi}{\partial Y}(t, Y) \approx \frac{V_{i+1}^{k}-V_{i-1}^{k}}{2 \delta y}
$$

Para calcularmos a magnitude do erro envolvido nesta aproximação, vamos utilizar expansões em séries de Taylor em torno dos nós $(t, Y+\delta y)$ e $(t, Y-\delta y)$ :

$$
\begin{aligned}
& \Phi(t, Y+\delta y)=\Phi(t, Y)+\delta y \frac{\partial \Phi}{\partial y}(t, Y)+\frac{1}{2} \delta y^{2} \frac{\partial^{2} \Phi}{\partial Y^{2}}(t, Y)+O\left(\delta y^{3}\right) \\
& \Phi(t, Y-\delta y)=\Phi(t, Y)-\delta y \frac{\partial \Phi}{\partial y}(t, Y)+\frac{1}{2} \delta y^{2} \frac{\partial^{2} \Phi}{\partial Y^{2}}(t, Y)+O\left(\delta y^{3}\right)
\end{aligned}
$$

Subtraindo a segunda expansão da primeira, e dividindo ambos os lados da equação por $2 \delta y$, obtemos

$$
\frac{\Phi(t, Y+\delta y)-\Phi(t, Y-\delta y)}{2 \delta y}=\frac{\partial \Phi}{\partial Y}(t, Y)+O\left(\delta y^{2}\right)
$$


o que mostra que a magnitude do erro introduzido por esta aproximação é da ordem de $\delta y^{2}$.

Esta aproximação é valida apenas para $1 \leq i \leq I-1$, pois, para $i=0$, não conhecemos $V_{i-1}^{k}$ e, para $i=I$, não conhecemos $V_{i+1}^{0}$. A solução deste problema depende das características da opção que estamos utilizando. No caso da opção com preço de exercício fixo (fixed strike call), vamos utilizar

$$
\begin{gathered}
V_{0}^{k}=2 V_{1}^{k}-V_{2}^{k} \mathrm{e} \\
V_{I}^{k}=V_{I-1}^{k} .
\end{gathered}
$$

No primeiro caso $\left(V_{0}^{k}\right)$, estamos forçando que, no limite inferior do intervalo de integração de $Y$, a segunda derivada da função em relação a $Y$ seja zero, ou seja, que gamma seja zero. Isto se deve ao fato de que, para valores grandes de $Y$, o payoff da opção é uma função aproximadamente linear de $Y$, o que implica em

$$
\lim _{Y \rightarrow-\infty} \frac{\partial^{2} \Phi}{\partial Y^{2}}=0
$$

Já no segundo caso $\left(V_{I}^{k}\right)$, estamos forçando que, no limite superior do intervalo de integração de $Y$, a primeira derivada da função em relação a $Y$ seja zero, ou seja, que delta seja zero, indicando que uma pequena variação em $Y$ não altera o valor da opção.

Finalmente, a aproximação que utilizaremos para $\frac{\partial^{2} \Phi}{\partial Y^{2}}$ será

$$
\frac{\partial^{2} \Phi}{\partial Y^{2}}(t, Y) \approx \frac{V_{i+1}^{k}-2 V_{i}^{k}+V_{i-1}^{k}}{\delta y^{2}},
$$


o que também introduz erros da ordem de $\delta y^{2}$, como pode ser deduzido somando as duas séries de Taylor em torno dos nós $(t, Y+\delta y)$ e $(t, Y-\delta y)$,

$$
\Phi(t, Y+\delta y)+\Phi(t, Y-\delta y)=2 \Phi(t, Y)+\delta y^{2} \frac{\partial^{2} \Phi}{\partial Y^{2}}(t, Y)+O\left(\delta y^{3}\right)
$$

dividindo ambos os lados por $\delta y^{2}$, e rearranjando, obtendo

$$
\frac{\partial^{2} \Phi(t, Y)}{\partial Y^{2}}=\frac{\Phi(t, Y+\delta y)-2 \Phi(t, Y)+\Phi(t, Y-\delta y)}{\delta y^{2}}+O\left(\delta y^{2}\right)
$$

Como na aproximação de $\frac{\partial \Phi}{\partial Y}$, este cálculo é válido apenas para $1 \leq i \leq I-1$, e considerações semelhantes podem ser feitas para os casos em que $i=0$ e $i=I$.

Agora, podemos reescrever a EDP de maneira apropriada para a resolução pelo método de diferenças finitas explícito:

$$
V_{i}^{k} r+\frac{V_{i}^{k}-V_{i}^{k+1}}{\delta t}-\frac{V_{i+1}^{k}-V_{i-1}^{k}}{2 \delta y}\left(\frac{1}{T}+r Y\right)+\frac{1}{2}\left(\frac{V_{i+1}^{k}-2 V_{i}^{k}+V_{i-1}^{k}}{\delta y^{2}}\right) \sigma^{2} Y^{2}=0
$$

A idéia do método é determinar $V_{i}^{k+1}$ a partir de $V_{i-1}^{k}, V_{i}^{k}$ e $V_{i+1}^{k}$, isto é:

$$
V_{i}^{k+1}=A_{i}^{k} V_{i-1}^{k}+B_{i}^{k} V_{i}^{k}+C_{i}^{k} V_{i+1}^{k} .
$$

Com algumas manipulações na fórmula da EDP, obtemos:

$$
A_{i}^{k}=\frac{1}{2}\left(\sigma^{2} Y^{2} \frac{\delta t}{\delta y^{2}}+\left(\frac{1}{T}+r Y\right) \frac{\delta t}{\delta y}\right)
$$




$$
\begin{aligned}
& B_{i}^{k}=1+r \delta t-\sigma^{2} Y^{2} \frac{\delta t}{\delta y^{2}}, \mathrm{e} \\
& C_{i}^{k}=\frac{1}{2}\left(\sigma^{2} Y^{2} \frac{\delta t}{\delta y^{2}}-\left(\frac{1}{T}+r Y\right) \frac{\delta t}{\delta y}\right) .
\end{aligned}
$$

A equação acima é válida para $1 \leq i \leq I-1$. A determinação de $V_{0}^{k}$ e $V_{I}^{k}$, a cada passo $k$, é feita substituindo-se os termos como explicado anteriormente:

$$
\begin{gathered}
V_{0}^{k}=2 V_{1}^{K}-V_{2}^{K} \mathrm{e} \\
V_{I}^{k}=V_{I-1}^{k} .
\end{gathered}
$$

\subsection{Resultados Obtidos}

Aplicando o método descrito na seção anterior para resolver a EDP relevante para o nosso problema e calcular o preço de uma opção asiática de compra com preço de exercício fixo (fixed strike), obtivemos os resultados das tabelas 2.1, 2.2 e 2.3 abaixo. Em todas elas, assumimos $S(0)=100$ e $T=1$, de forma a obter resultados compatíveis com os obtidos por Rogers e Shi (1995). Além disso, adotamos $\lambda=2$ para o fator de amplitude da quantidade $Y$, após testes empíricos com diferentes valores de $\lambda$, o que significa que $Y$ varia no intervalo $\left[-\frac{X}{S_{0}}, 3 \frac{X}{S_{0}}\right]$. 


\begin{tabular}{|c|c|c|c|c|c|}
\hline \\
\hline \multicolumn{6}{|c|}{$\begin{array}{l}\text { Tabela } 2.1 \text { - Opçăo asiática com preço de exercício fixo } \\
\text { T }\end{array}$} \\
\hline \multirow{2}{*}{\multicolumn{6}{|c|}{$\begin{array}{r}100 \\
0.05\end{array}$}} \\
\hline SIGMA & 0,05 & & & & \\
\hline$I$ & Excercicio & Re $S$ & $I_{1}$ & $I_{2}$ & $I_{3}$ \\
\hline \multirow[t]{6}{*}{0,05} & 95 & 7,157 & 6,994 & 7,202 & 7,174 \\
\hline & & $(29,4)$ & $(2,9)$ & $(23,7)$ & $(79,3)$ \\
\hline & 100 & 2,621 & 1,197 & 2,368 & 2,584 \\
\hline & & $(29,4)$ & $(2,9)$ & 23,21 & $(78,3)$ \\
\hline & 105 & 0,439 & 0,112 & 0,238 & 0,286 \\
\hline & & $(29,4)$ & $(2,9)$ & $(23,2)$ & $\{81,0)$ \\
\hline \multirow[t]{6}{*}{0,09} & 95 & 8,823 & 10,176 & 8,791 & 8,808 \\
\hline & & $(31,3)$ & $(2,9)$ & $(23,4)$ & $(78,7)$ \\
\hline & 100 & 4,185 & 2,552 & 4,137 & 4,245 \\
\hline & & $(31,3)$ & $(2,9)$ & $(24,5)$ & $(78,3)$ \\
\hline & 105 & 1,011 & 0,312 & 0,702 & 0,836 \\
\hline & & $(31,3)$ & $(2,9)$ & $27,2\}$ & $(79,5)$ \\
\hline \multirow[t]{6}{*}{0,15} & 95 & 11,090 & 12,703 & 11,104 & 11,093 \\
\hline & & $(45,0)$ & $(2,9)$. & $(24,5)$ & $(78,3)$ \\
\hline & 100 & 6,777 & 6,216 & 6,833 & 6,789 \\
\hline & & $(45,0)$ & $(3,0)$ & $(23,3)$ & $(81,1)$ \\
\hline & 105 & 2,639 & 1,179 & 2,379 & 2,608 \\
\hline & & $(45,0)$ & $(3,2)$ & $23,7)$ & $(81,3)$ \\
\hline
\end{tabular}

\begin{tabular}{|c|c|c|c|c|c|}
\hline \multicolumn{6}{|c|}{ Tabela 2.2 - Opção asiática com preço de exercicio fixo } \\
\hline $\mathbf{T}$ & & & & & \\
\hline $\mathbf{s}_{0}$ & 100 & & & & \\
\hline SIGMA & 0,10 & & & & \\
\hline 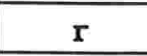 & Exereício & Re S & $I_{1}$ & $\mathbf{I}_{2}$ & $\mathbf{I}_{3}$ \\
\hline 0,05 & 90 & 11,942 & 11,930 & 11,944 & 11,948 \\
\hline & & $(41, x)$ & $(11,8)$ & $(96,8)$ & $(323,7)$ \\
\hline & 100 & 3,624 & 3,446 & 3,594 & 3,620 \\
\hline & & $(41,1)$ & $(11,8)$ & $(95,4)$ & $(330,9)$ \\
\hline & 110 & 0,359 & 0,342 & 0,333 & 0,332 \\
\hline & & $(41,1)$ & $(11,9)$ & $(108,5)$ & $(323,2)$ \\
\hline 0,09 & 90 & 13,382 & 13,376 & 13,382 & 13,384 \\
\hline & & $(44,6)$ & $(12,2)$ & $(99,2)$ & $(323,8)$ \\
\hline & 100 & 4,887 & 4,714 & 4,868 & 4,895 \\
\hline & & $(44,6)$ & $(12,5)$ & $(98,5)$ & $(322,1)$ \\
\hline & 110 & 0,659 & 0,613 & 0,624 & 0,627 \\
\hline & & $(44,6)$ & $(12,2)$ & $(98,4)$ & $(324,0)$ \\
\hline 0,15 & 90 & 15,398 & 15,395 & 15,397 & 15,398 \\
\hline & & $(55,4)$ & $(13,7)$ & $(98,4)$ & $(3 \times 5,7)$ \\
\hline & 100 & 7,000 & 6,879 & 6,994 & 7,013 \\
\hline & & $(55,4)$ & $(11,8)$ & $(97,6)$ & $(325,7)$ \\
\hline & 110 & 1,430 & 1,319 & 1,387 & 1,401 \\
\hline & & $(55,4)$ & $(11,8)$ & $(100,4)$ & $(325,3)$ \\
\hline
\end{tabular}




\begin{tabular}{|c|c|c|c|c|c|}
\hline $\begin{array}{l}\text { Tabela } \\
T\end{array}$ & .3 - Opção & 20 & $\mathrm{pr}$ & ex & 80 \\
\hline $\mathrm{s}_{0}$ & 100 & & & & \\
\hline SIGMA & 0,20 & & & & \\
\hline$\Gamma$ & Exercício & $R$ e $S$ & $I_{1}$ & $I_{2}$ & $I_{3}$ \\
\hline 0,05 & 90 & 12,589 & 12,570 & 12,590 & 12,593 \\
\hline & & $(84,3)$ & $(46,1)$ & $(370,2)$ & $(1249,7)$ \\
\hline & 100 & 5,760 & 5,733 & 5,755 & 5,760 \\
\hline & & $(84,3)$ & $(46,1)$ & $(370,3)$ & $(1249,1)$ \\
\hline & 110 & 1,996 & 1,987 & 1,989 & 1,989 \\
\hline & & $(84,3)$ & $(46,1)$ & $(369, y)$ & $(1250,0)$ \\
\hline 0,09 & 90 & 13,825 & 13,809 & 13,826 & 13,829 \\
\hline & & $(88,1)$ & $(46,2)$ & $(370,1)$ & $(1250,4)$ \\
\hline & 100 & 6,773 & 6,744 & 6,769 & 6,774 \\
\hline & & 8 & $(49,3)$ & $(369,3)$ & $(1251,6)$ \\
\hline & 110 & 2,551 & 2,536 & 2,544 & 2,545 \\
\hline & & $(88, y)$ & $(49,7)$ & $(370,7)$ & $(1250,5)$ \\
\hline 0,15 & 90 & 15,636 & 15,624 & 15,637 & 15,640 \\
\hline & & $(102,0)$ & $(48,7)$ & $(369,1)$ & $(1250,0)$ \\
\hline & 100 & 8,402 & 8,373 & 8,400 & 8,405 \\
\hline & & $(102,0)$ & $(46,8)$ & $(370,5)$ & $(1249,4)$ \\
\hline & 110 & 3,558 & 3,535 & 3,550 & 3,553 \\
\hline & & $(100,0)$ & $(46,7)$ & $(870,7)$ & $(1250,0)$ \\
\hline
\end{tabular}

As colunas exibidas nas tabelas são:

$\mathrm{r}$ - taxa de juros livre de risco;

Exercício - preço de exercício da opção;

R e S - melhores resultados obtidos por Rogers e Shi (1995);

$I_{1}, I_{2}$ e $I_{3}$ - resultados obtidos com 500, 1000 e 1500 subintervalos para Y, respectivamente.

Os valores exibidos entre parênteses se referem ao tempo, em segundos, demandado para cada cálculo. Cabe ressaltar que os tempos nas colunas $I_{1}, I_{2}$ e $I_{3}$ servem apenas de referência para comparações entre si, e não são comparáveis com os tempos obtidos por Rogers e Shi (1995), cujos cálculos foram efetuados em computadores de características diferentes.

Observando-se os resultados obtidos, verificamos que, para $\sigma=0,05$, os valores não são bons, apresentando em alguns casos grandes variações em relação aos resultados obtidos 
por Rogers e Shi (1995). Já para valores maiores de $\sigma$, no entanto, os resultados por eles obtidos são muito próximos aos nossos. Com $\sigma=0,20$ e 1500 subintervalos (coluna $\mathrm{I}_{3}$ da Tabela 2.3), por exemplo, as diferenças entre os dois valores são em todos os casos inferiores a $0,4 \%$. Já os valores obtidos na Tabela 2.2 situam-se num patamar intermediário em relação a essas diferenças. Estas constatações parecem indicar que o algoritmo que construímos é tanto melhor quanto maior é a volatilidade do ativo-base. As mesmas observações se aplicam à taxa de juros, indicando também que, de modo geral, o algoritmo fornece respostas melhores para taxas de juro maiores. Observa-se também que a qualidade das respostas piora significativamente para preços de exercício maiores do que o preço do ativo-objeto, o que pode indicar que a definição do intervalo de variação de $Y$ não funciona bem para estes casos.

Com relação ao tempo de cálculo, observamos em todas as tabelas que, quando dobramos o número de subintervalos da quantidade $Y$, o tempo de cálculo aumenta em cerca de oito vezes. Além disso, comparando as tabelas entre si, observamos que, para o mesmo número de subintervalos, quando dobramos o valor da volatilidade do ativo-base, o tempo de cálculo aumenta em cerca de quatro vezes.

A obtenção de valores mais precisos pode ser conseguida através do aumento do número de subintervalos da quantidade $Y$, mas esta técnica, em contrapartida, eleva substancialmente o tempo demandado para o cálculo, como pode ser observado comparando-se as colunas $\mathrm{I}_{1}$, $\mathrm{I}_{2}$ e $\mathrm{I}_{3}$ de cada tabela.

Todas estas observações estão relacionadas às condições de estabilidade do método utilizado, que, utilizando a abordagem descrita por Wilmott (2000), são:

$$
\begin{gathered}
r \geq 0, \\
\delta t \leq \frac{1}{\sigma^{2} I^{2}} \mathrm{e} \\
\delta y \leq \frac{\sigma^{2} I}{r} .
\end{gathered}
$$


A primeira condição é satisfeita por definição, pois a taxa de juros livre de risco é sempre positiva. A terceira condição deve ser satisfeita pela escolha adequada de $I$, que é o número de passos da quantidade $Y$. Cabe notar que, quanto menor a volatilidade do ativoobjeto em relação à taxa de juros livre de risco, maior tem que ser a escolha de $I$, aumentando também o tempo de cálculo da EDP. Mas, se ambos forem da ordem de $10^{-1}$, que é uma situação bastante comum nos mercados financeiros, o número de passos da quantidade $Y$ deve ser no mínimo da ordem de $10^{2}$, o que não implica em nenhuma restrição séria do ponto de vista do tempo de cálculo. Já a segunda condição, uma restrição sobre $\delta t$, exerce o efeito de aumentar demasiadamente o tempo de cálculo do valor desejado, a cada tentativa de melhora da precisão do cálculo. Por exemplo, supondo que partimos de um cálculo com baixa precisão, isto é, com $\delta y$ relativamente grande, podemos querer melhorar a precisão reduzindo $\delta y$ à metade. Neste caso, pela segunda condição de estabilidade, somos obrigados a reduzir $\delta t$ a $1 / 4$ do valor inicial, o que implica em aumentar em cerca de oito vezes o tempo de cálculo. A precisão, no entanto, irá melhorar apenas por um fator de quatro vezes, pois a magnitude do erro se reduz a

$$
O\left(1 / 4 \delta t,(1 / 2 \delta y)^{2}\right)=\frac{1}{4} O\left(\delta t, \delta y^{2}\right)
$$

Estas constatações em relação a $\delta t, \delta y$ e ao tempo de cálculo foram verificadas empiricamente nas tabelas apresentadas acima. Por exemplo, na Tabela 2.1, este é provavelmente o motivo pelo qual o resultado obtido para $r=0,05$ e Exercício $=100$, mesmo com o maior número de subintervalos (coluna $\mathrm{I}_{3}$ ), não é satisfatório.

A discussão acima deixa bastante claro que o método apresentado impõe limitações à questão da melhora da precisão versus o aumento do tempo demandado para o cálculo. Tais limitações motivam a pesquisa e implementação de outros métodos de apreçamento de opções asiáticas, que possam resolver de maneira mais satisfatória esta questão. Este é o assunto do próximo capítulo, onde desenvolveremos um outro método para o apreçamento de opções asiáticas. 


\section{DETERMIINAÇÃO DE LIMITES PARA O PREÇO DA OPÇÃO}

O cálculo do preço de uma opção asiática através da resolução numérica de uma EDP pode ser um processo excessivamente demorado em termos computacionais, como foi visto no capítulo anterior, caso a precisão exigida na resposta seja grande. Muito embora seja possível melhorar significativamente a magnitude dos erros envolvidos através da utilização de métodos mais sofisticados para a resolução da $\mathrm{EDP}^{1}$, e apesar do constante aprimoramento observado nos computadores ao longo dos anos, ainda assim o tempo computacional demandado pode comprometer a sua utilização prática em ambientes como uma mesa de operações de um grande banco, onde as exigências com relação à precisão, ao tempo de resposta e à quantidade de cálculos diferentes são muito grandes.

Uma tentativa de conciliar estas questões, melhorando a relação entre a precisão e o tempo de cálculo, é a determinação de um intervalo suficientemente pequeno que contenha o preço da opção, através de um método suficientemente rápido em termos computacionais, e que não envolva a resolução de uma EDP. Este é o objetivo deste capítulo, e o que vamos determinar é um limite inferior para o preço da opção, e o erro máximo em relação a este preço. Através da soma destes dois valores, obtemos também um limite superior para o preço da opção. Vamos começar enunciando dois lemas, que vão orientar o desenvolvimento de nosso objetivo:

Lema 3.1: Para quaisquer duas variáveis aleatórias $Y$ e $Z$,

$$
\mathrm{E}\left(Y^{+}\right) \geq \mathrm{E}\left(\mathrm{E}(Y \mid Z)^{+}\right)
$$

Prova: Pela Lei das Esperanças Iteradas, temos que $\mathrm{E}\left(Y^{+}\right)=\mathrm{E}\left(\mathrm{E}\left(Y^{+} \mid Z\right)\right)$. Vamos então verificar a variável aleatória $\mathrm{E}\left(Y^{+} \mid Z\right)$ :

\footnotetext{
${ }^{1}$ Um exemplo de método mais sofisticado para a resolução numérica de uma EDP é o método de CrankNicolson, cuja magnitude do erro é $O\left(\delta t^{2}, \delta y^{2}\right)$. Veja Wilmott (2000) para mais detalhes.
} 


$$
\begin{aligned}
\mathrm{E}\left(Y^{+} \mid Z\right) & =\int_{-\infty}^{+\infty} y^{+} f_{Y \mid Z}(y) d y \\
& =\int_{0}^{+\infty} y f_{Y \mid Z}(y) d y \\
& =\left(\int_{0}^{+\infty} y f_{Y \mid Z}(y) d y\right)^{+} \\
& \geq\left(\int_{-\infty}^{0} y f_{Y \mid Z}(y) d y+\int_{0}^{+\infty} y f_{Y \mid Z}(y) d y\right)^{+} \\
& =\left(\int_{-\infty}^{+\infty} y f_{Y \mid Z}(y) d y\right)^{+} \\
& =\mathrm{E}(Y \mid Z)^{+}
\end{aligned}
$$

Tomando a esperança de ambos os lados, e utilizando novamente a Lei das Esperanças Iteradas, obtemos

$$
\mathrm{E}\left(\mathrm{E}\left(Y^{+} \mid Z\right)\right)=\mathrm{E}\left(Y^{+}\right) \geq \mathrm{E}\left(\mathrm{E}(Y \mid Z)^{+}\right)
$$

Lema 3.2: Para quaisquer duas variáveis aleatórias $Y$ e $Z$,

$$
0 \leq \mathrm{E}\left(\mathrm{E}\left(Y^{+} \mid Z\right)\right)-\mathrm{E}\left(\mathrm{E}(Y \mid Z)^{+}\right) \leq \frac{1}{2} \mathrm{E}\left(\operatorname{Var}(Y \mid Z)^{1 / 2}\right) .
$$

Prova: Do lema anterior, segue imediatamente que $0 \leq \mathrm{E}\left(\mathrm{E}\left(Y^{+} \mid Z\right)\right)-\mathrm{E}\left(\mathrm{E}(Y \mid Z)^{+}\right)$. Agora, fazendo $\mathrm{E}\left(Y^{+} \mid Z\right)=\mathrm{E}\left(U^{+}\right)$e $\mathrm{E}(Y \mid Z)^{+}=\mathrm{E}(U)^{+}$, temos

$$
\begin{aligned}
& \mathrm{E}(U)=\mathrm{E}(U)^{+}+\mathrm{E}(U)^{-} \\
& \mathrm{E}(|U|)=\mathrm{E}(U)^{+}-\mathrm{E}(U)^{-} .
\end{aligned}
$$

Somando as duas equações, obtemos

$$
\mathrm{E}(U)+\mathrm{E}(|U|)=2 \mathrm{E}(U)^{+}
$$


Mas

$$
\begin{gathered}
\mathrm{E}(U) \geq 0 \Rightarrow \mathrm{E}(U)=\mathrm{E}(U)^{+}=|\mathrm{E}(U)| \\
\mathrm{E}(U)<0 \Rightarrow \mathrm{E}(U)^{+}=0 \text { e } \mathrm{E}(U)=-|\mathrm{E}(U)| .
\end{gathered}
$$

Logo, vale a relação

$$
\mathrm{E}(U)=2 \mathrm{E}(U)^{+}-|\mathrm{E}(U)| \cdot
$$

Substituindo (II) em (I), e rearranjando, obtemos

$$
\mathrm{E}\left(U^{+}\right)-\mathrm{E}(U)^{+}=\frac{1}{2}(\mathrm{E}(|U|)-|\mathrm{E}(U)|)
$$

Agora, pela desigualdade triangular, e utilizando $\mathrm{E}(|U|)=|\mathrm{E}(|U|)|$, temos que

$$
\frac{1}{2}(\mathrm{E}(|U|)-|\mathrm{E}(U)|) \leq \frac{1}{2} \mathrm{E}(|U-\mathrm{E}(U)|)=\frac{1}{2} \mathrm{E}\left(\operatorname{Var}(U)^{1 / 2}\right),
$$

que, desfazendo as substituições anteriores $\left(\mathrm{E}\left(U^{+}\right)=\mathrm{E}\left(Y^{+} \mid Z\right)\right.$ e $\left.\mathrm{E}(U)^{+}=\mathrm{E}(Y \mid Z)^{+}\right)$, nos fornece

$$
\mathrm{E}\left(\mathrm{E}\left(Y^{+} \mid Z\right)\right)-\mathrm{E}\left(\mathrm{E}(Y \mid Z)^{+}\right) \leq \frac{1}{2} \mathrm{E}\left(\operatorname{Var}(Y \mid Z)^{1 / 2}\right)
$$

Aplicando estes dois lemas ao nosso problema, podemos estimar um limite inferior para o preço da opção (Lema 3.1), e também o erro máximo, ou diferença máxima, entre o limite inferior e o preço real da opção (Lema 3.2). A idéia é condicionar a variável aleatória $Y$, cuja esperança queremos calcular, a uma outra variável aleatória $Z$ qualquer, e tomar a esperança de $Y$ através do cálculo da esperança condicional sob todos os valores de $Z$. 
Nossa escolha de $Z$, levando em conta a facilidade de cálculo de sua esperança $(\mathrm{E}(Z))$ e variância $\left(\mathrm{E}\left((Z-\mathrm{E}(Z))^{2}\right)\right)$, bem como do termo cruzado $\mathrm{E}(Y Z)$, será $Z=\int_{0}^{1} B_{u} d u$. Temos então que:

$$
\begin{gathered}
\mathrm{E}(Z)=0 ; \\
\mathrm{E}\left((Z-\mathrm{E}(Z))^{2}\right)=\mathrm{E}\left(Z^{2}\right)=\mathrm{E}\left(\int_{0}^{1} B_{u} d u \int_{0}^{1} B_{s} d s\right)=\int_{0}^{1} \int_{0}^{1} \mathrm{E}\left(B_{u} B_{s}\right) d u d s ; \\
\text { e } \mathrm{E}\left(B_{t} Z\right)=\mathrm{E}\left(B_{t} \int_{0}^{1} B_{u} d u\right)=\int_{0}^{1} \mathrm{E}\left(B_{t} B_{u}\right) d u .
\end{gathered}
$$

E, como

$$
\mathrm{E}\left(B_{u} B_{s}\right)=\left\{\begin{array}{l}
\mathrm{E}\left(B_{u}\left(B_{u}+\left(B_{s}-B_{u}\right)\right)\right)=\mathrm{E}\left(B_{u}^{2}\right)=u, u \leq s \\
\mathrm{E}\left(B_{s}\left(B_{s}+\left(B_{u}-B_{s}\right)\right)\right)=\mathrm{E}\left(B_{s}^{2}\right)=s, u>s
\end{array}\right\}=\min (s, u),
$$

obtemos

$$
\mathrm{E}\left(Z^{2}\right)=\int_{0}^{1} \int_{0}^{1} \min (s, u) d u d s=\int_{0}^{1}\left(\int_{0}^{s} u d u+\int_{s}^{1} s d u\right) d s=\int_{0}^{1} \frac{s^{2}}{2}+s(1-s) d s=\frac{1}{3}
$$

e, de forma equivalente

$$
\mathrm{E}\left(B_{t} Z\right)=\int_{0}^{1} \min (t, u) d u=\int_{0}^{t} u d u+\int_{t}^{1} t d u=\frac{t^{2}}{2}+t(1-t)=\frac{t(2-t)}{2} .
$$

Na próxima seção, vamos aplicar estes resultados ao nosso problema, para determinar o limite inferior que procuramos. 


\subsection{Obtenção de Limite Inferior para o Preço da Opção}

$\mathrm{O}$ que queremos determinar é um limite inferior para $\mathrm{E}\left(\mathrm{Y}^{+}\right)$, que, como já vimos, é o mesmo que $\mathrm{E}\left(\mathrm{E}(Y \mid Z)^{+}\right)$, para qualquer variável aleatória $Z$. Fazendo $Z=\int_{0}^{1} B_{u} d u$, temos

$$
\mathrm{E}(Y \mid Z)=\mathrm{E}\left(\int_{0}^{1} \exp \left(\sigma B_{t}-\frac{1}{2} \sigma^{2} t+r t\right) d t \mid Z\right)=\int_{0}^{1} e^{-\frac{1}{2} \sigma^{2} t+r t} \mathrm{E}\left(e^{\sigma B_{t}} \mid Z\right) d t
$$

Mas

$$
\begin{aligned}
\mathrm{E}\left(e^{\sigma B_{t}} \mid Z\right) & =\int_{-\infty}^{+\infty} e^{\sigma b} f_{B \mid Z}(b \mid z) d b \\
& =\int_{-\infty}^{+\infty} e^{\sigma b}\left(\frac{f(b, z)}{\int_{-\infty}^{+\infty} f(b, z) d b}\right) d b \\
& =\frac{\int_{-\infty}^{+\infty} e^{\sigma b} f(b, z) d b}{\int_{-\infty}^{+\infty} f(b, z) d b}
\end{aligned}
$$

onde $f_{B \mid Z}(b z)$ é a distribuição condicional de $B_{t}$ dado $Z$, e $f(b, z)$ é a distribuição conjunta de $B_{t}$ e $Z$.

Como $B_{t} \sim \mathrm{N}(0, t)$ e $Z \sim \mathrm{N}(0,1 / 3)$ são variáveis aleatórias gaussianas, a sua distribuição conjunta $f(b, z)$ é uma distribuição gaussiana bivariada, cuja fórmula geral é dada por

$$
f(b, z)=\frac{1}{2 \pi \sigma_{b} \sigma_{z} \sqrt{1-\rho^{2}}} \exp \left(-\frac{1}{2\left(1-\rho^{2}\right)}\left(\left(\frac{b-\mu_{b}}{\sigma_{b}}\right)^{2}-2 \frac{\rho\left(b-\mu_{b}\right)\left(z-\mu_{z}\right)}{\sigma_{b} \sigma_{z}}+\left(\frac{z-\mu_{z}}{\sigma_{z}}\right)^{2}\right)\right),
$$

onde 


$$
\begin{aligned}
& \mu_{b}=\mu_{z}=0, \\
& \sigma_{b}=\sqrt{t} \mathrm{e} \\
& \sigma_{z}=\frac{1}{\sqrt{3}}, \\
& \sigma_{b z}=\mathrm{E}(b z)=\frac{t(2-t)}{2}, \mathrm{e} \\
& \rho=\frac{\sigma_{b z}}{\sigma_{b} \sigma_{z}}=\frac{t(2-t)}{2} \frac{\sqrt{3}}{\sqrt{t}}=\frac{\sqrt{3 t}(2-t)}{2} .
\end{aligned}
$$

Então, introduzindo estes valores na fórmula original, obtemos

$$
\begin{aligned}
f(b, z) & =\frac{1}{2 \pi \sqrt{t / 3 \sqrt{1-\frac{3 t(2-t)^{2}}{4}}}} \exp \left(\frac{-1}{2\left(1-\frac{3 t(2-t)^{2}}{4}\right)}\left(\frac{b^{2}}{t}-2 \frac{\sqrt{3 t}(2-t)}{2} \frac{\sqrt{3}}{\sqrt{t}} b z+3 z^{2}\right)\right) \\
& =\frac{\sqrt{3}}{2 \pi \sqrt{t-3 t^{2}+3 t^{3}-3 / 4 t^{4}}} \exp \left(\frac{-1}{2-6 t+6 t^{2}-3 / 2 t^{3}}\left(\frac{b^{2}}{t}-6 b z+3 b z t+3 z^{2}\right)\right)
\end{aligned}
$$

$\mathrm{E}$, substituindo na fórmula anterior, para $\mathrm{E}\left(e^{\sigma B_{t}} \mid Z\right)$, obtemos

$$
\begin{aligned}
\mathrm{E}\left(e^{\sigma B_{t}} \mid Z\right)= & \frac{\int_{-\infty}^{+\infty} e^{\sigma b}\left(\frac{\sqrt{3}}{2 \pi \sqrt{t-3 t^{2}+3 t^{3}-3 / 4 t^{4}}}\right) \exp \left(\frac{-1}{2-6 t+6 t^{2}-3 / 2 t^{3}}\left(\frac{b^{2}}{t}-6 b z+3 b z t+3 z^{2}\right)\right) d b}{\int_{-\infty}^{+\infty}\left(\frac{\sqrt{3}}{2 \pi \sqrt{t-3 t^{2}+3 t^{3}-3 / 4 t^{4}}}\right) \exp \left(\frac{-1}{2-6 t+6 t^{2}-3 / 2 t^{3}}\left(\frac{b^{2}}{t}-6 b z+3 b z t+3 z^{2}\right)\right) d b} \\
= & \frac{\int_{-\infty}^{+\infty} \exp \left(\sigma b+\frac{-1}{2-6 t+6 t^{2}-3 / 2 t^{3}}\left(\frac{b^{2}}{t}-6 b z+3 b z t+3 z^{2}\right)\right) d b}{\int_{-\infty}^{+\infty} \exp \left(\frac{-1}{2-6 t+6 t^{2}-3 / 2 t^{3}}\left(\frac{b^{2}}{t}-6 b z+3 b z t+3 z^{2}\right)\right) d b}=\frac{A}{B}, \text { onde }
\end{aligned}
$$




$$
\begin{gathered}
A=\int_{-\infty}^{+\infty} \exp \left(\sigma b+\frac{-1}{2-6 t+6 t^{2}-3 / 2 t^{3}}\left(\frac{b^{2}}{t}-6 b z+3 b z t+3 z^{2}\right)\right) d b \mathrm{e} \\
B=\int_{-\infty}^{+\infty} \exp \left(\frac{-1}{2-6 t+6 t^{2}-3 / 2 t^{3}}\left(\frac{b^{2}}{t}-6 b z+3 b z t+3 z^{2}\right)\right) d b .
\end{gathered}
$$

Calculando estas duas integrais (utilizamos o software matemático Maple 6), obtemos

$$
\begin{aligned}
& A=\frac{\sqrt{2 \pi t\left(4-12 t+12 t^{2}-3 t^{3}\right)}}{2} \exp \left(-\frac{3}{8} \sigma^{2} t^{4}+\frac{3}{2} \sigma^{2} t^{3}-\frac{3}{2} \sigma t^{2} z-\frac{3}{2} \sigma^{2} t^{2}+3 \sigma t z+\frac{1}{2} \sigma^{2} t-\frac{3}{2} z^{2}\right) \\
& \text { e } \\
& B=\frac{\sqrt{2 \pi t\left(4-12 t+12 t^{2}-3 t^{3}\right)}}{2} \exp \left(-\frac{3}{2} z^{2}\right) .
\end{aligned}
$$

Então

$$
\begin{aligned}
\mathrm{E}\left(e^{\sigma B_{t}} \mid Z\right) & =\frac{A}{B} \\
& =\exp \left(-\frac{3}{8} \sigma^{2} t^{4}+\frac{3}{2} \sigma^{2} t^{3}-\frac{3}{2} \sigma t^{2} z-\frac{3}{2} \sigma^{2} t^{2}+3 \sigma t z+\frac{1}{2} \sigma^{2} t-\frac{3}{2} z^{2}+\frac{3}{2} z^{2}\right) \\
& =\exp \left(\sigma\left(-\frac{3}{2} t^{2}+3 t\right) z-\sigma^{2}\left(\frac{3}{8} t^{4}-\frac{3}{2} t^{3}+\frac{3}{2} t^{2}-\frac{1}{2} t\right)\right)
\end{aligned}
$$

e, finalmente,

$$
\begin{aligned}
\mathrm{E}(Y \mid Z) & =\int_{0}^{1} \exp \left(-\frac{1}{2} \sigma^{2} t+r t\right) \mathrm{E}\left(e^{\sigma B} \mid Z\right) d t \\
& =\int_{0}^{1} \exp \left(-\frac{1}{2} \sigma^{2} t+r t\right) \exp \left(\sigma\left(-\frac{3}{2} t^{2}+3 t\right) z-\sigma^{2}\left(\frac{3}{8} t^{4}-\frac{3}{2} t^{3}+\frac{3}{2} t^{2}-\frac{1}{2} t\right)\right) d t \\
& =\int_{0}^{1} \exp \left(\sigma \frac{3 t(2-t)}{2} z-\sigma^{2}\left(\frac{1}{2} t+\frac{3}{8} t^{4}-\frac{3}{2} t^{3}+\frac{3}{2} t^{2}-\frac{1}{2} t\right)+r t\right) d t \\
& =\int_{0}^{1} \exp \left(\sigma \frac{3 t(2-t)}{2} z-\frac{1}{6} \sigma^{2}\left(\frac{3 t(2-t)}{2}\right)^{2}+r t\right) d t
\end{aligned}
$$


Logo, podemos escrever o limite inferior que definimos para o preço da opção como

$$
\begin{aligned}
\mathrm{E}\left(\mathrm{E}(Y \mid Z)^{+}\right) & =\mathrm{E}\left(\left[\int_{0}^{1} \exp \left(\sigma \frac{3 t(2-t)}{2} z-\frac{1}{6} \sigma^{2}\left(\frac{3 t(2-t)}{2}\right)^{2}+r t\right) d t\right]^{+}\right) \\
& =\int_{-\infty}^{+\infty}\left[\int_{0}^{1} \exp \left(\sigma \frac{3 t(2-t)}{2} z-\frac{1}{6} \sigma^{2}\left(\frac{3 t(2-t)}{2}\right)^{2}+r t\right) d t\right]^{+} d z .
\end{aligned}
$$

Agora, aplicando o Lema 3.2, sabemos que a diferença entre o limite inferior, que acabamos de escrever em forma não estocástica, e o preço verdadeiro da opção está limitado a

$$
\mathrm{E}\left(Y^{+}\right)-\mathrm{E}\left(\mathrm{E}(Y \mid Z)^{+}\right) \leq \frac{1}{2} \mathrm{E}\left(\operatorname{Var}(Y \mid Z)^{1 / 2}\right)
$$

Lembrando que

$$
\operatorname{Var}(Y \mid Z)=\mathrm{E}\left(Y^{2} \mid Z\right)-(\mathrm{E}(Y \mid Z))^{2}
$$

e procedendo da mesma forma que na dedução de uma fórmula não estocástica para o limite inferior, obtemos

$$
\begin{aligned}
& \operatorname{Var}(Y \mid Z)=\int_{0}^{1} \int_{0}^{1} \exp \left[\sigma Z\left(\frac{3 t(2-t)}{2}+\frac{3 s(2-s)}{2}\right)-\frac{1}{2} \sigma^{2} \frac{1}{3}\left(\left(\frac{3 s(2-s)}{2}\right)^{2}+\left(\frac{3 t(2-t)}{2}\right)^{2}\right)+r s+r t\right] \\
& \exp \left[\sigma^{2}\left(\min (s, t)-\frac{3 s t(2-s)(2-t)}{4}\right)-1\right] d s d t \text {. }
\end{aligned}
$$

Conseqüentemente, a fórmula para a diferença máxima entre $\mathrm{E}\left(Y^{+}\right)$e $\mathrm{E}\left(\mathrm{E}(Y \mid Z)^{+}\right)$fica 


$$
\begin{array}{r}
\frac{1}{2} \mathrm{E}\left(\operatorname{Var}(Y \mid Z)^{1 / 2}\right)=\frac{1}{2} \int_{-\infty}^{+\infty}\left[\int_{0}^{1} \int_{0}^{1} \exp \sigma Z\left(\frac{3 t(2-t)}{2}+\frac{3 s(2-s)}{2}\right)-\frac{1}{2} \sigma^{2} \frac{1}{3}\left(\left(\frac{3 s(2-s)}{2}\right)^{2}+\left(\frac{3 t(2-t)}{2}\right)^{2}\right)+r s+r t\right] \\
\left.\exp \left[\sigma^{2}\left(\min (s, t)-\frac{3 s t(2-s)(2-t)}{4}\right)-1\right] d s d t\right]^{1 / 2} d z .
\end{array}
$$

Resta-nos, agora, calcular numericamente as expressões acima. Este é o assunto da próxima seção.

\subsection{Obtenção Numérica do Limite}

Para o cálculo dos valores encontrados na seção anterior, faz-se necessário implementar um método numérico de cálculo de uma integral em um intervalo. Escolhemos para esta tarefa o Método de Simpson, que é um dos mais utilizados, e também entre os mais fáceis de implementar. Este método aproxima o valor da integral de uma função $f(x)$ em um intervalo $[a, b]$ por polinômios de grau 2 , da seguinte forma:

- divide o intervalo $[a, b]$ em $n$ subintervalos ( $n$ par) de mesmo tamanho $h=\frac{b-a}{n}$;

- a cada dois intervalos contíguos, $[a+(i-1) h, a+i h]$ e $[a+i h, a+(i+1) h]$, $i=1 \ldots n-1$, define um polinômio de grau 2 que passa pelos três pontos $(a+(i-1) h, f(a+(i-1) h)), \quad(a+i h, f(a+i h))$ e $(a+(i+1) h, f(a+(i+1) h)), \quad$ e calcula a integral deste polinômio no intervalo $[a+(i-1) h, a+(i+1) h]$;

- a aproximação da integral de $f(x)$ é dada pela soma das integrais obtidas no passo anterior em cada par de subintervalos.

A fórmula de integração obtida por este método é:

$$
\int_{a}^{b} f(x)=\frac{1}{3}(f(a)+4 f(a+h)+2 f(a+2 h)+\cdots+4 f(a+(n-2) h)+2 f(a+(n-1) h)+f(b)) .
$$


O erro $(\varepsilon)$ envolvido nesta aproximação é dado por

$$
\varepsilon \leq \frac{h^{5}}{90} \max _{x \in[a, b]}\left|f^{(4)}(x)\right|
$$

No cálculo do limite inferior, a função a ser integrada em relação a $t$ é

$$
f(t, z)=\exp \left(\sigma \frac{3 t(2-t)}{2} z-\frac{1}{6} \sigma^{2}\left(\frac{3 t(2-t)}{2}\right)^{2}+r t\right)
$$

que é crescente em $t, z, \mathrm{e} r$, e a função a ser integrada em $z$ é

$$
g(z)=\exp \left(-3 z^{2}\left[\int_{0}^{1} f(t, z)\right]\right)
$$

Assumindo $r=0,5$ e $\sigma=0,8$, calculando a quarta derivada de $f(t, z)$ e, em seguida, a quarta derivada de $g(z)$, para valores de $z$ entre -10 e 10, obtivemos o gráfico da Figura 3.1, que mostra valores muito altos nas proximidades de $z=0$.

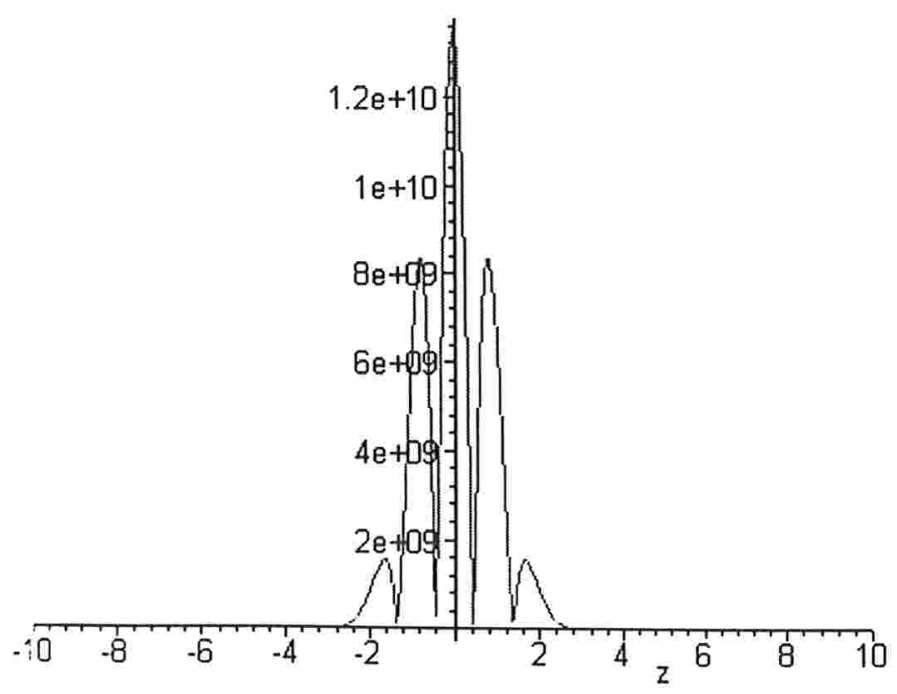

Figura 3.1 - Gráfico de $\left|g^{(4)}(z)\right|$. 
Assumindo então o valor de $2 \mathrm{E} 10$ para $\left|g^{(4)}(z)\right|$, obtemos a estimativa para o erro

$$
\varepsilon \leq \frac{h^{5}}{90} \max _{x \in[a, b]}\left|f^{(4)}(x)\right| \leq \frac{h^{5}}{90} 2 \times 10^{10},
$$

que, se escolhermos $h=\frac{1}{200}$, nos leva a

$$
\varepsilon \leq \frac{(1 / 200)^{5}}{90} 2 \times 10^{10} \cong 0,0007
$$

um valor que podemos desprezar, considerando a precisão que queremos obter.

\subsection{Resultados Obtidos}

Os resultados obtidos para o limite inferior do preço da opção, bem como para a diferença máxima entre o limite inferior e o preço da opção, estão exibidos nas tabelas 3.1, 3.2 e 3.3. Da mesma forma que nos resultados obtidos na resolução da EDP, efetuada no capítulo anterior, aqui também assumimos $S(0)=100$ e $T=1$, de forma a obter resultados comparáveis com os obtidos por Rogers e Shi (1995). Todos os cálculos foram feitos considerando opções asiáticas de compra com preço de exercício fixo (fixed strike). 


\begin{tabular}{|c|c|c|c|c|c|c|c|c|}
\hline $\begin{array}{l}\text { Tabela } \\
\text { T } \\
\text { So } \\
\text { SIGMA }\end{array}$ & $\begin{array}{r}3.1-\text { Opçă } \\
1 \\
100 \\
0,05\end{array}$ & 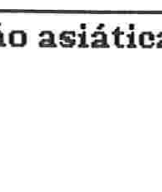 & on $\mathrm{p}^{\mathrm{x}}$ & e & 10 fisko & & & \\
\hline $\boldsymbol{r}$ & Exercicio & EDP RS & EDP & Linf RS & Lsup RS & Linf & Dif Max & Lsup \\
\hline 0,05 & 95 & 7,157 & 7,174 & 7,178 & 7,183 & 7,178 & 0,001 & 7,179 \\
\hline & & $(29,4)$ & $(79,3)$ & $(2,95)$ & $(0,6 y)$ & $(0,33)$ & $(317,3)$ & \\
\hline & 100 & 2,621 & 2,584 & 2,716 & 2,722 & 2,714 & 0,001 & 2,715 \\
\hline & & $(29,4)$ & $(78,3)$ & $R, 95)$ & $(0,61)$ & $(0,22)$ & $(317,3)$ & \\
\hline & 105 & 0,439 & 0,286 & 0,337 & 0,343 & 0,336 & 0,001 & 0,337 \\
\hline & & $(29,4)$ & $(8 x, 0)$ & $2,95)$ & $(0,61)$ & $(0,17)$ & $(317,3)$ & \\
\hline 0,09 & 95 & 8,823 & 8,808 & 8,809 & 8,821 & 8,809 & 0,002 & 8,810 \\
\hline & & $(31,3)$ & $(78,7)$ & {$[2,96)$} & $(0,56)$ & $(0,16)$ & $(319,1)$ & \\
\hline & 100 & 4,185 & 4,245 & 4,308 & 4,318 & 4,307 & 0,002 & 4,309 \\
\hline & & $(3 x, 3)$ & $(78,3)$ & $R, 961$ & $(0,56)$ & $(0,22)$ & $(319,1)$ & \\
\hline & 105 & 1,011 & 0,836 & 0,958 & 0,968 & 0,957 & 0,002 & 0,958 \\
\hline & & $(31,3)$ & $(79,5)$ & $(2,96)$ & $(0,56)$ & $(0,17)$ & $(319,1)$ & \\
\hline 0,15 & 95 & 11,090 & 11,093 & 11,094 & 11,114 & 11,094 & 0,002 & 11,097 \\
\hline & & $(45,0)$ & $(78,3)$ & $p, 961$ & $(0,59)$ & $(0,16)$ & $(3 \pm 7,3)$ & \\
\hline & 100 & 6,777 & 6,789 & 6,794 & 6,810 & 6,794 & 0,002 & 6,797 \\
\hline & & $(45,0)$ & $(81,1)$ & $(2,96)$ & $(0,59)$ & $(0,16)$ & $(317,3)$ & \\
\hline & 105 & 2,639 & 2,608 & 2,744 & 2,761 & 2,744 & 0,002 & 2,746 \\
\hline & & $(45,0)$ & $(81,3)$ & $(2,96)$ & $(0,59)$ & $(0,23)$ & $(3 \geq 7,3)$ & \\
\hline
\end{tabular}

\begin{tabular}{|c|c|c|c|c|c|c|c|c|}
\hline $\begin{array}{l}\text { Tabela } \\
\text { T } \\
\text { So } \\
\text { SIGMA }\end{array}$ & $\begin{array}{r}3.2-0 p c \\
1 \\
100 \\
0,10\end{array}$ & asiat & 2 & 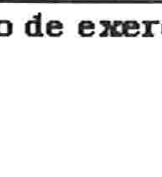 & 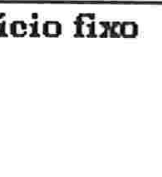 & & & \\
\hline$r$ & Exoercício & EDP RS & EDP & Linf RS & Lsup RS & Linf & Dif Max & Lsup \\
\hline 0,05 & 90 & $\begin{array}{c}11,942 \\
(41,1)\end{array}$ & $\begin{array}{c}11,948 \\
(323,7)\end{array}$ & $\begin{array}{c}11,951 \\
0,67\end{array}$ & & 11,951 & 0,003 & 11,954 \\
\hline & 100 & $\begin{array}{l}3,624 \\
(41, x)\end{array}$ & $\begin{array}{c}3,620 \\
(330,9)\end{array}$ & $\begin{array}{l}3,641 \\
2,67\end{array}$ & $\begin{array}{l}3,663 \\
(0,56)\end{array}$ & $\begin{array}{l}3,645 \\
0,17)\end{array}$ & 0,003 & 3,648 \\
\hline & 110 & $\begin{array}{l}0,359 \\
\{41,1\}\end{array}$ & $\begin{array}{r}0,332 \\
(323,2)\end{array}$ & $\begin{array}{l}0,331 \\
2,67)\end{array}$ & $\begin{array}{l}0,353 \\
(0,56)\end{array}$ & $\begin{array}{r}0,328 \\
(0,22) \\
\end{array}$ & $\begin{array}{l}0,003 \\
(318,7)\end{array}$ & 0,331 \\
\hline 0,09 & 90 & $\begin{array}{c}13,382 \\
(44,6)\end{array}$ & $\begin{array}{l}13,384 \\
(323,8)\end{array}$ & $\begin{array}{c}13,385 \\
(3, \infty 2)\end{array}$ & $\begin{array}{c}13,410 \\
(0,57)\end{array}$ & $\begin{array}{c}13,385 \\
(0,16)\end{array}$ & $\begin{array}{l}0,004 \\
(3 \times 7,6)\end{array}$ & 13,389 \\
\hline & 100 & $\begin{array}{l}4,887 \\
(44,6)\end{array}$ & $\begin{array}{l}4,895 \\
822, y\end{array}$ & 4,915 & 4,942 & 4,918 & 0,004 & 4,922 \\
\hline & 110 & 0,659 & $\begin{array}{c}0,627 \\
(324,0)\end{array}$ & $\begin{array}{l}(3, \infty 2) \\
0,630 \\
(3, \infty 2)\end{array}$ & $\begin{array}{l}(0,57) \\
0,657 \\
(0,57)\end{array}$ & $\begin{array}{l}(0,17) \\
0,625 \\
(0,22)\end{array}$ & $\begin{array}{c}(317,6) \\
0,004 \\
(317,6)\end{array}$ & 0,630 \\
\hline 0,15 & 90 & $\begin{array}{c}15,398 \\
(55,4)\end{array}$ & $\begin{array}{l}15,398 \\
(315,7)\end{array}$ & $\begin{array}{c}15,399 \\
(3,14)\end{array}$ & $\begin{array}{c}15,445 \\
(0,56)\end{array}$ & $\begin{array}{c}15,399 \\
(0,16)\end{array}$ & $\begin{array}{c}0,006 \\
\beta \geq 7,3\rangle\end{array}$ & 15,405 \\
\hline & 100 & $\begin{array}{l}7,000 \\
(55,4)\end{array}$ & $\begin{array}{c}7,013 \\
(325,7)\end{array}$ & $\begin{array}{l}7,028 \\
(3,14)\end{array}$ & $\begin{array}{l}7,066 \\
0,56)\end{array}$ & $\begin{array}{l}7,027 \\
(0,17)\end{array}$ & $\begin{array}{l}0,006 \\
(317,3)\end{array}$ & 7,033 \\
\hline & 110 & $\begin{array}{r}1,430 \\
(55,4) \\
\end{array}$ & $\begin{array}{c}1,401 \\
(325,3)\end{array}$ & $\begin{array}{r}1,413 \\
(3,14)\end{array}$ & $\begin{array}{r}1,451 \\
\mid 0,56)\end{array}$ & $\begin{array}{r}1,415 \\
(0,22)\end{array}$ & $\begin{array}{r}0,006 \\
(317,3)\end{array}$ & 1,421 \\
\hline
\end{tabular}




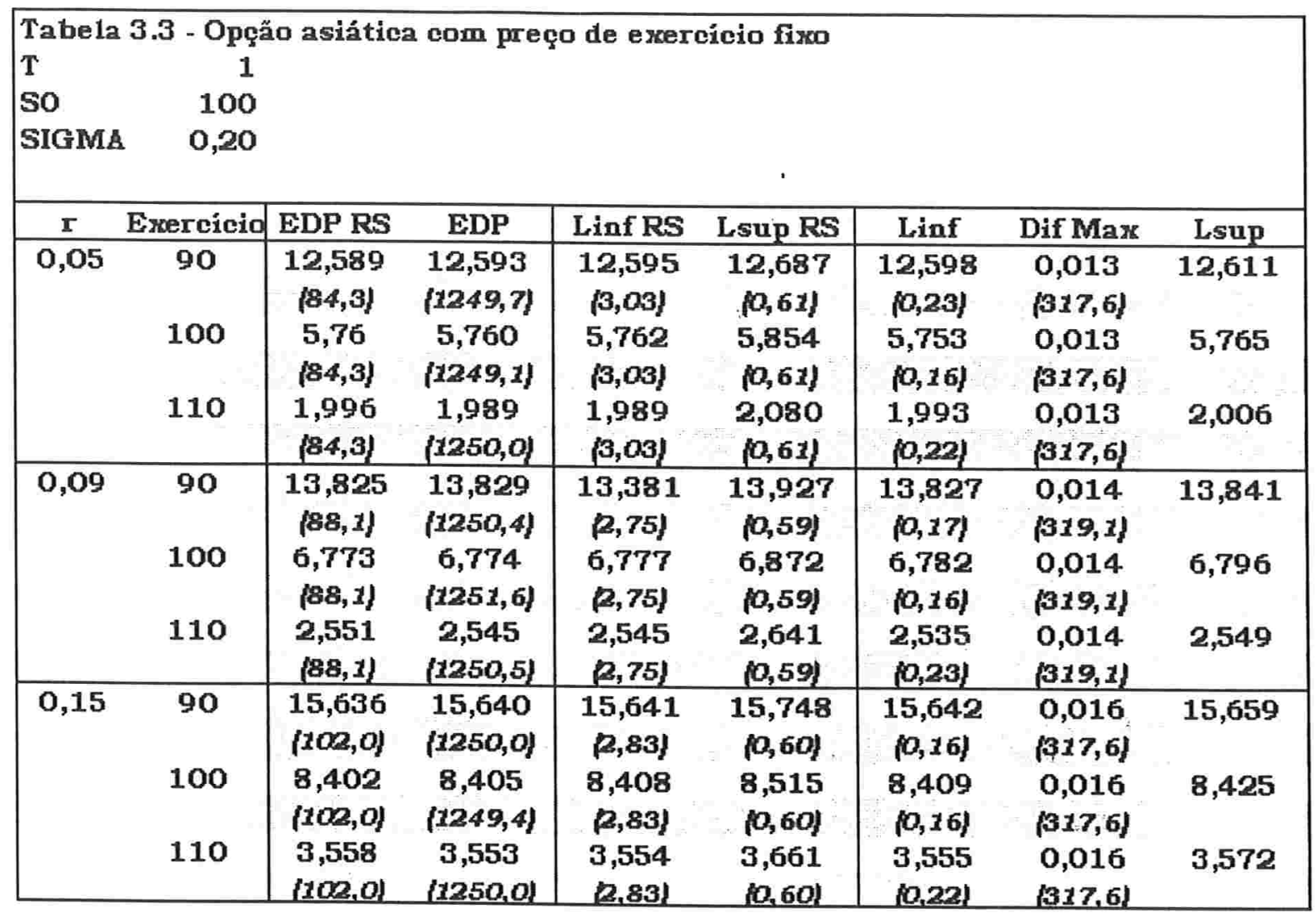

As colunas exibidas nas tabelas são:

$\mathrm{r}$ - taxa de juros livre de risco;

Exercício - preço de exercício da opção;

EDP RS - melhor resultado obtido por Rogers e Shi (1995) através da resolução da EDP;

EDP - melhor resultado que obtivemos através da resolução da EDP (coluna $\mathrm{I}_{3}$ nas tabelas do capítulo anterior);

Linf RS e Lsup RS - limite inferior e limite superior, respectivamente, obtidos por Rogers e Shi (1995);

Linf - limite inferior obtido através do método descrito neste capítulo;

Dif Max - valor obtido para a diferença máxima (ou erro máximo) entre o limite inferior e o preço da opção;

Lsup - limite superior obtido através do método descrito neste capítulo. 
Os valores exibidos entre parênteses se referem ao tempo, em segundos, demandado para cada cálculo. Como ressaltado no capítulo anterior, os tempos que obtivemos não são comparáveis com os tempos obtidos por Rogers e Shi (1995), cujos cálculos foram efetuados em computadores de características diferentes. Optamos por apresentar em colunas separadas a diferença máxima e o limite superior, diferentemente dos resultados apresentados por Rogers e Shi (1995), para ressaltar a magnitude do primeiro.

Com relação aos limites inferiores que obtivemos, podemos observar, nas três tabelas, que eles estão muito próximos aos obtidos por Rogers e Shi (1995). Além disso, como também aconteceu no artigo citado, estes valores, em muitos casos, são maiores dơ que os que foram obtidos através do cálculo da EDP. A análise sobre a magnitude dos erros envolvidos nos cálculos da EDP e do limite inferior nos leva a concluir que o limite inferior provavelmente aproxima melhor o preço da opção, por envolver erros de menor magnitude. Por outro lado, os erros da EDP podem ser reduzidos através do aumento do número de subintervalos utilizados, como vimos no capítulo anterior, mas a um custo alto do ponto de vista do tempo demandado para o cálculo.

O tempo necessário para calcular a diferença máxima pelo método que descrevemos nas seções anteriores é bastante superior ao tempo de cálculo do limite inferior, ao contrário dos resultados apresentados por Rogers e Shi (1995). Esta diferença se deve a uma série de aproximações que aqueles autores fazem, reduzindo significativamente o tempo de cálculo, mas com conseqüências sobre a precisão do resultado obtido, como pode ser observado nas tabelas que apresentamos. No entanto, como o cálculo em questão não depende da volatilidade do ativo-base, observamos que, nas situações em que esta última é relativamente alta, o tempo demandado é inferior ao que é necessário para o cálculo da EDP.

Nossa conclusão, após as considerações expostas acima, é que a determinação do preço da opção através do cálculo de uma EDP pode ser substituída, com nítidas vantagens do ponto de vista do tempo de cálculo, pela determinação do limite inferior para este preço, conforme descrito neste capítulo. Os valores que obtivemos para a diferença máxima 
envolvida nesta aproximação, em todos os casos apresentados, não são superiores a $1 \%$, sendo provavelmente inferiores aos erros na estimação da volatilidade do ativo-base, e indicando que, do ponto de vista prático, a determinação do limite inferior é suficiente. 


\section{UMA APLICAÇÃO AO MERCADO BRASILEIRO DE TAXAS DE CÂMBIO}

Como introdução ao assunto que discutiremos neste capítulo, vamos explorar algumas características das opções asiáticas e, quando pertinente, suas diferenças em relação às opções européias tradicionais.

Já vimos anteriormente que as opções asiáticas têm seu payoff determinado pela média dos preços do ativo-base durante um certo período da vida da opção. Além disso, elas podem ser definidas e utilizadas sobre qualquer ativo-base (financeiro ou não), desde que seja possível a coleta dos preços deste nas datas definidas para a formação da média. Alguns exemplos de ativos-base que podem ser utilizados são índices de ações, taxas de juros, taxas de câmbio e commodities. Assim como para uma opção européia tradicional, a característica fundamental do ativo-base no processo de apreçamento da opção asiática (além, claro, de seu preço corrente) é sua volatilidade, embora o efeito desta não seja o mesmo nos dois casos. Para entender esta relação, vamos explorar a relação entre o preço do ativo-base e a sua média.

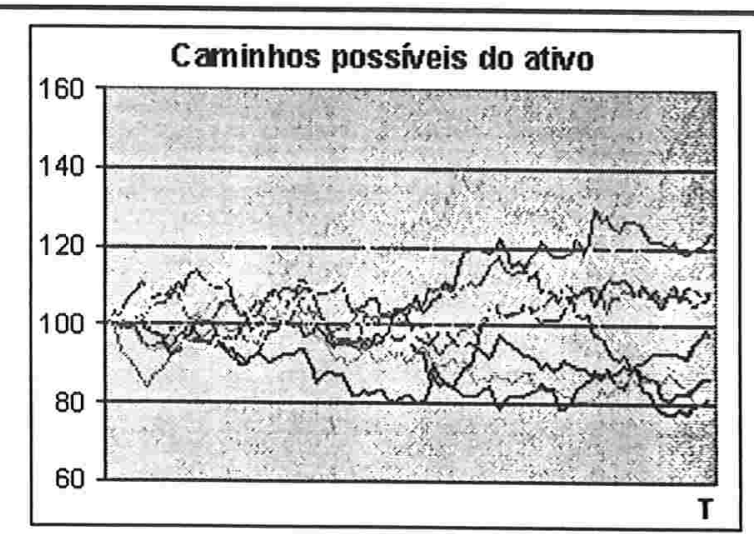

(a) ex emplos de caminhos percorridos por um ativo

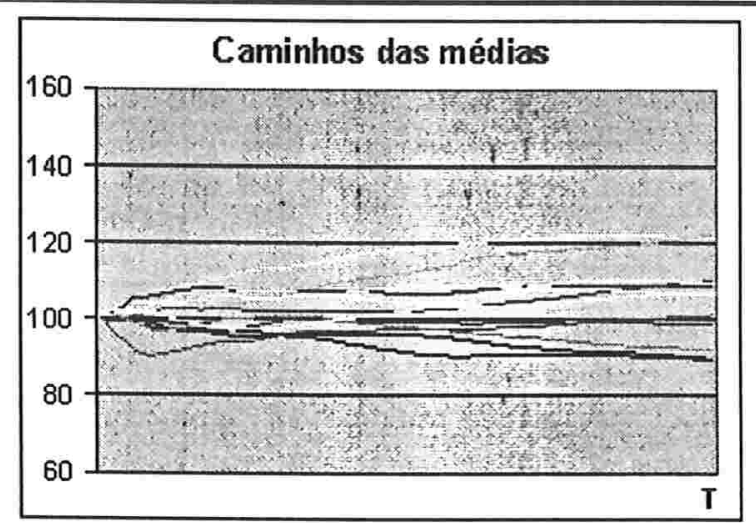

(b) caminhos das médias correspondentes

Figura 4.1: Comparação entre a evolução dos preços de um ativo e a evolução de sua média.

Como vimos no Exemplo 1.1, o preço inicial da opção asiática é sempre menor do que o preço inicial de uma opção européia tradicional com as mesmas características (mesmo preço de exercício, mesma data de maturidade e sobre o mesmo ativo-base) $)^{2}$, justamente porque, no lançamento da opção, a esperança do payoff da opção asiática é menor do que a 
esperança do payoff da opção européia correspondente. Na Figura 4.1(a), apresentamos graficamente alguns caminhos que podem ser traçados pelo ativo-base até a maturidade e, na Figura 4.1(b), os caminhos traçados pelas respectivas médias aritméticas. Além de verificar que as médias exibem uma variação menor do que os preços, é possível observar também que as variações das médias são menos bruscas do que as variações dos preços, indicando que a volatilidade da média também é menor do que a volatilidade dos preços.

Por outro lado, se observarmos a evolução da média através do tempo, veremos que a influência da volatilidade nesta evolução é significativa (vide Figura 4.2). Isto se deve ao fato de que um ativo com volatilidade alta exibe grandes variações de preço e, como conseqüência, a evolução temporal da média de seus preços também tende a ser mais volátil.

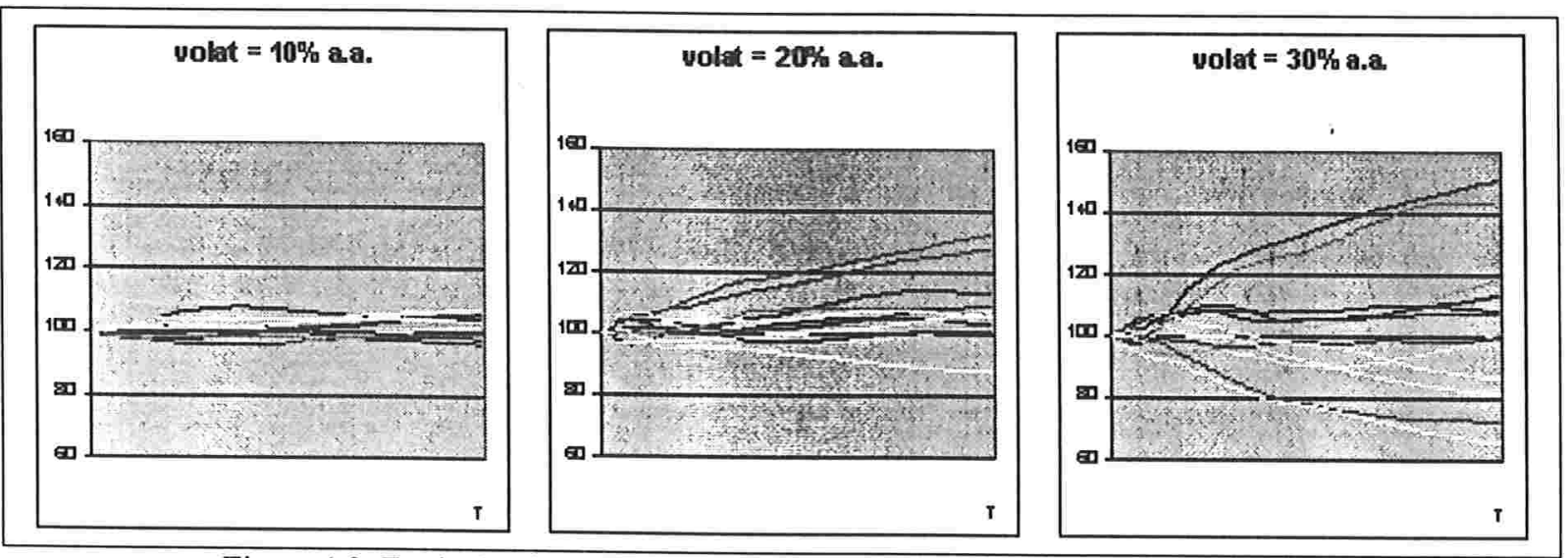

Figura 4.2: Evolução da média com diferentes volatilidades para o preço do ativo.

Também já vimos, durante o desenvolvimento dos dois métodos para apreçamento das opções asiáticas, que os outros fatores que influenciam seu preço são o tempo a decorrer até a maturidade, o preço de exercício e a taxa de juros livre de risco. A taxa de juros livre de risco exerce uma influência mais sutil na média: quando ela é relativamente alta, além de também fazer com que a evolução temporal da média experimente maiores variações (embora com intensidade menor do que no caso da volatilidade), exerce o efeito de mudar a inclinação da média, como pode ser observado na Figura 4.3.

\footnotetext{
${ }^{2}$ Embora, em alguns casos especiais citados no Capitulo 1, estes dois preços possam ser iguais.
} 


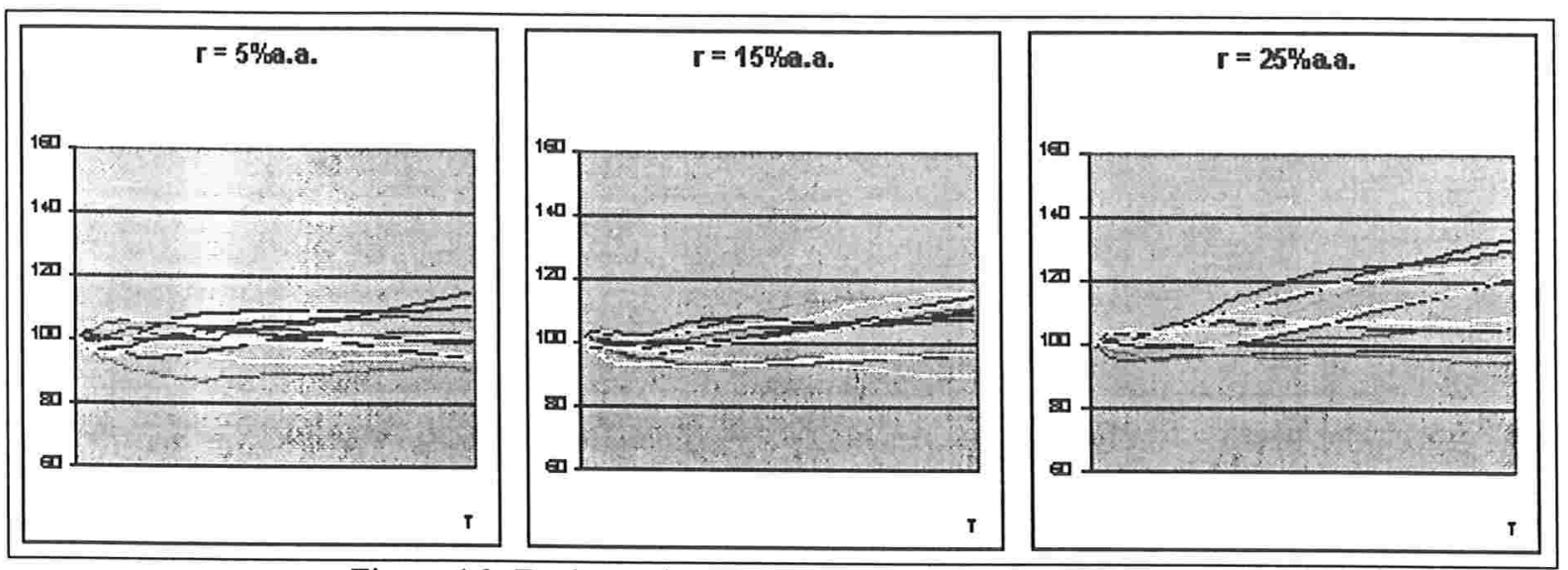

Figura 4.3: Evolução da média com diferentes taxas de juros.

Embora os exemplos exibidos nas figuras acima tenham sido elaborados com médias aritméticas e pesos constantes, as observações são válidas também para médias geométricas e pesos não constantes.

Vejamos agora o comportamento dos preços das opções asiáticas de compra, com preço de exercício fixo e média aritmética, comparando-os com os preços de opções européias equivalentes. Nas tabelas abaixo, apresentamos uma aplicação prática em que o ativo-base é a taxa de câmbio de reais por dólar. Assumimos que a taxa corrente é $R \$ 2.300,00$ por mil dólares e a maturidade de ambas as opções é um ano. Cada tabela corresponde a uma volatilidade anual diferente para a taxa de câmbio ( $20 \%, 30 \%$ e $50 \%$ respectivamente), e exibimos os valores iniciais obtidos para ambas as opções para diversos preços de exercício e taxas de juros. 


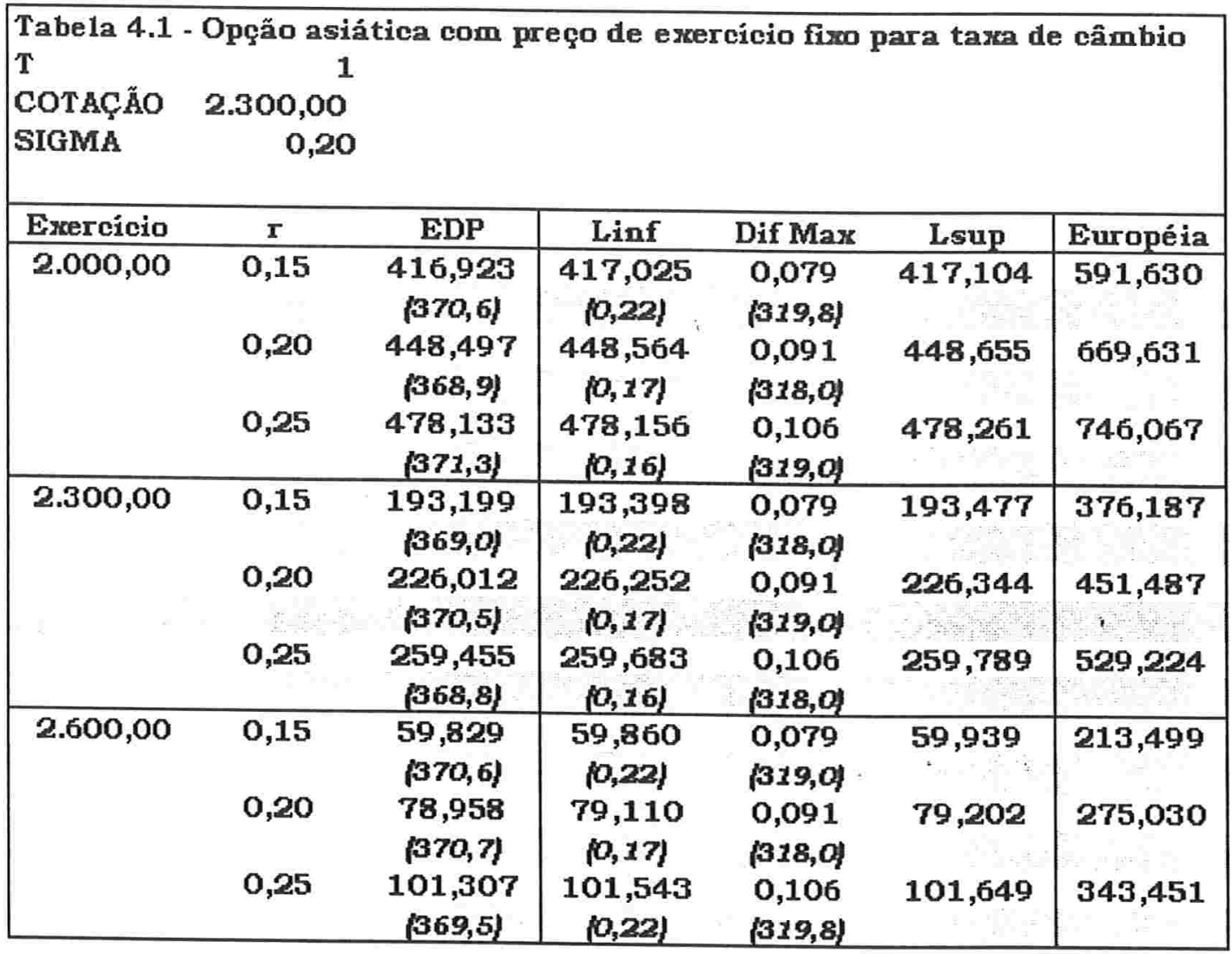

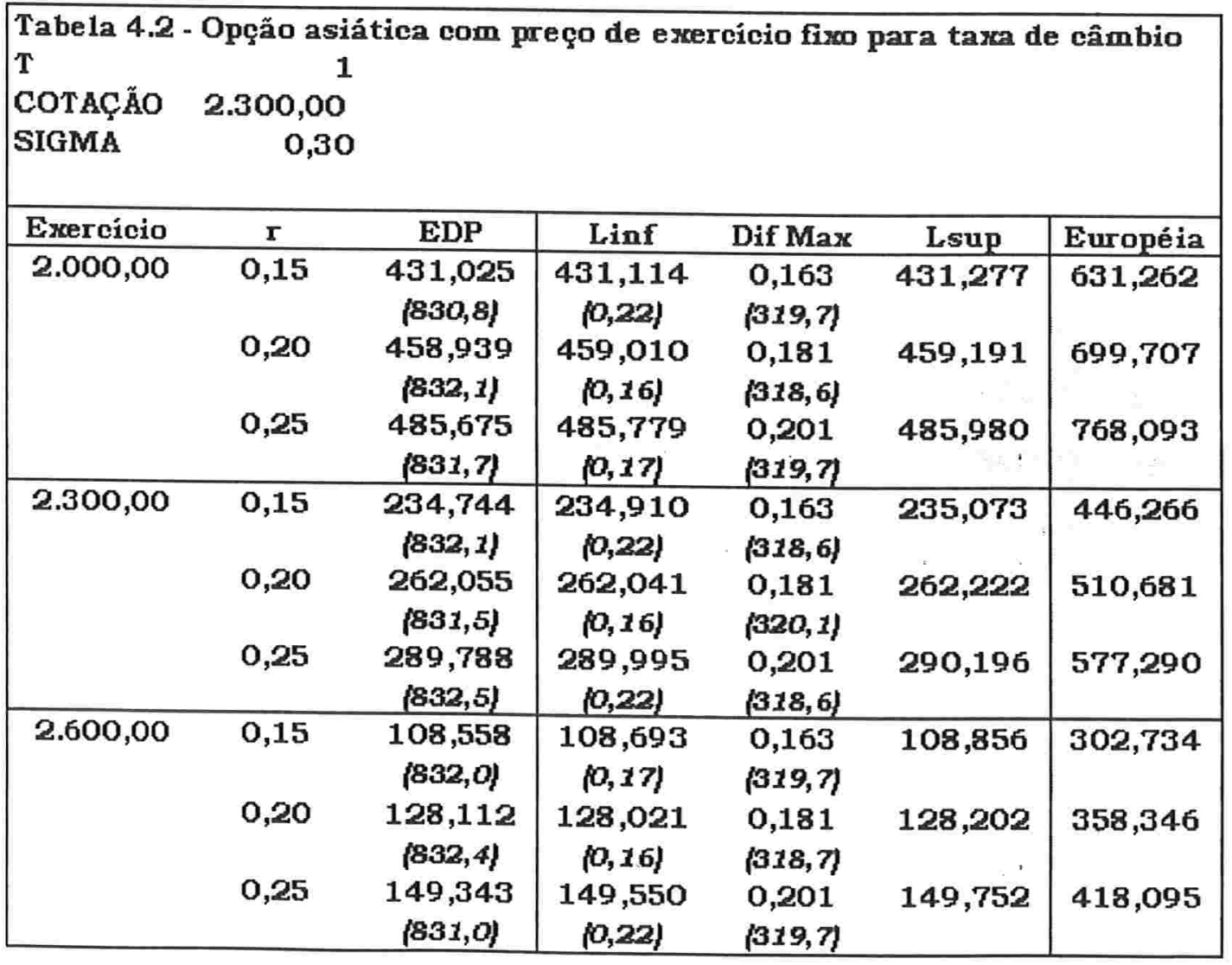




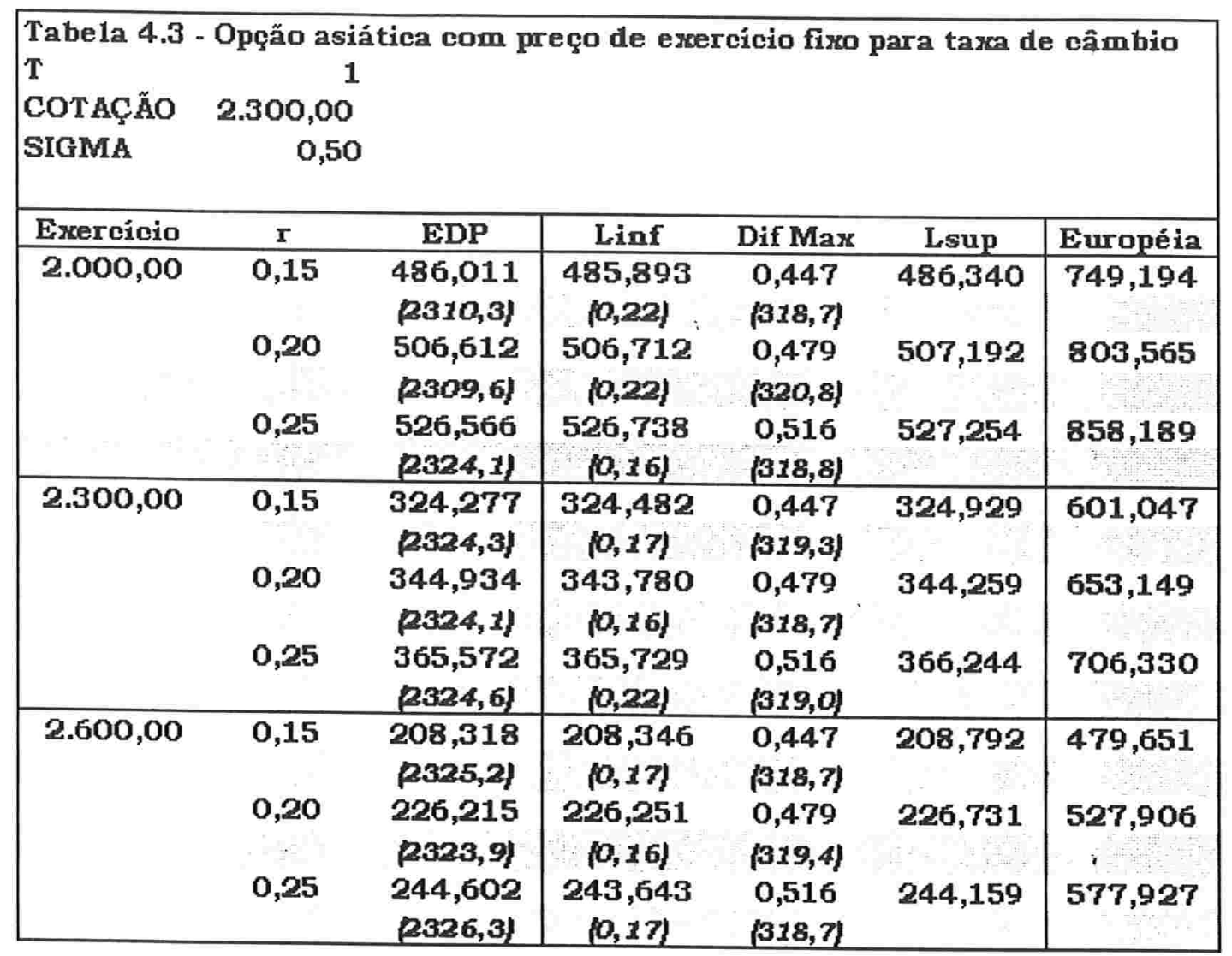

As colunas exibidas nas tabelas são:

Exercício - preço de exercício da opção;

$\mathrm{r}$ - taxa de juros livre de risco;

EDP - resultado que obtivemos através da resolução da EDP com 1000 subintervalos para a quantidade $Y$;

Linf - limite inferior obtido através do método descrito anteriormente;

Dif Max - valor obtido para a diferença máxima (ou erro máximo) entre o limite inferior e o preço da opção;

Lsup - limite superior obtido através do método descrito anteriormente;

Européia - valor da opção européia equivalente.

Os valores exibidos entre parênteses se referem ao tempo, em segundos, demandado para cada cálculo. 
Em todos os casos, o tempo demandado para o cálculo da diferença máxima é menor do que o demandado para o cálculo do preço através da resolução da EDP, e esta diferença aumenta para volatilidades maiores da taxa de câmbio, pelos mesmos motivos discutidos no capítulo anterior. Apesar disto, o tempo de cálculo das diferenças máximas é relativamente alto, se considerarmos as necessidades de uma mesa de operações de um grande banco. Por outro lado, também como já discutido no capítulo anterior, o valor obtido para estas diferenças é muito pequeno (sempre bem inferior a $1 \%$, nos casos apresentados), justificando o cálculo apenas dos limites inferiores, que é muito rápido, e a utilização destes valores como boas aproximações dos preços verdadeiros.

Comparando-se os preços obtidos para as opções asiáticas com os das européias, constatamos, nos casos apresentados, que as diferenças entre eles são maiores nos casos em que o preço de exercício é mais alto, situando-se algumas vezes em patamares superiores a $200 \%$. Mesmo nos casos em que as diferenças são menores, elas se situam em patamares altos, sempre superiores a $40 \%$. Os valores utilizados para todos os parâmetros são adequados à realidade brasileira, dando-nos uma idéia palpável da sensibilidade da utilização, na definição do payoff da opção, da média ao invés do preço do ativo-base na maturidade. 


\section{CONCLUSÕES}

Através da definição e do detalhamento de um método rápido e eficiente de apreçamento de opções asiáticas, pretendemos oferecer uma contribuição efetiva ao mercado financeiro brasileiro.

Vimos, no capítulo 2, uma abordagem para o apreçamento de opções asiáticas através da resolução numérica de uma PDE. O tempo computacional necessário para os cálculos, bem como a magnitude dos erros envolvidos, inviabilizam a utilização prática desta abordagem em um ambiente de negócios, onde respostas ágeis e precisas são essenciais.

Como alternativa a este problema, apresentamos, no capítulo 3, uma forma de determinar um intervalo suficientemente pequeno em torno do preço da opção. O limite inferior deste intervalo pode ser calculado quase instantaneamente, enquanto que, para o limite superior, o tempo demandado é ainda muito grande, em alguns casos até superior ao tempo de cálculo da PDE. Entretanto, com base na análise do erro envolvido no processo, e da magnitude da diferença entre o limite inferior e o preço da opção, argumentamos que, para os objetivos de um ambiente de negócios, é suficiente determinar o limite inferior.

Os exemplos apresentados no Capítulo 4, por si só, justificam a execução de estudos de viabilidade para a introdução sistemática das opções asiáticas no mercado financeiro brasileiro. Tal medida poderia vir a favorecer investidores em busca de proteção para seus ativos e passivos, por representar menores custos em relação às opções européias e, em muitos casos, uma proteção mais efetiva, como já comentamos na introdução deste trabalho. Os benefícios também se fariam sentir no mercado financeiro de maneira geral, na medida em que representaria uma alternativa a mais à disposição de todas as partes interessadas, colaborando com a melhora da liquidez dos mercados, e possivelmente trazendo novos participantes.

Possíveis extensões deste trabalho podem ser levadas a cabo em diversas direções. Os métodos aqui expostos podem ser estendidos, com poucas modificações, para os outros tipos de opções asiáticas definidos no capítulo 1, e que não foram abordados aqui. Pode-se 
também analisar a utilização de opções asiáticas sobre outros ativos-base, contemplando outros mercados e ampliando ainda mais a gama de possibilidades de utilização destas opções. Por fim, estudos de viabilidade visando à introdução destas opções no mercado devem incluir um aprofundamento das questões relativas à exata definição de quais preços, e com que periodicidade, poderiam ser utilizados no cálculo da média, levando em conta as limitações de ordem técnica e legal. 


\section{BIBLIOGRAFIA}

1. Baxter, Martin and Rennie, Andrew (1998) - Financial Calculus - An Introduction to Derivative Pricing, Cambridge University Press

2. Campbell, John Y., Lo, Andrew W. e MacKinlay, A. Craig (1997) - The Econometrics of Financial Markets, Princeton University Press

3. Hull, John C. (1999) - Options, Futures and Other Derivatives, Prentice Hall

4. Klebaner, Fima C. (1999) - Introduction to Stochastic Calculus with Applications, Imperial College Press

5. Neftci, Salih N. (1996) - An Introduction to the Mathematics of Financial Derivatives, Academic Press

6. Rogers, L.C.G. e Shi, Z. (1995) - The Value of an Asian Option, Journal of Applied Probability, 32 - pp. 1077-1088

7. Ross, Sheldon (1997) - A First Course in Probabilty, Prentice Hall

8. Taleb, Nassim (1997) - Dynamic Hedging - Managing Vanilla and Exotic Options, John Wiley \& Sons, Inc

9. Vorst, Tom C. F. (1990) - Averaging Options, in The Handbook of Exotic Options, ed. Israel Nelken, Irwin Professional Publishing

10. Wilmott, Paul (2000) - Paul Wilmott on Quantitative Finance Vol. 1 e 2, John Wiley \& Sons, Inc 